EUROPEAN ORGANISATION FOR NUCLEAR RESEARCH

CERN-EP/98-113

16th July 1998

\title{
Di-Jet Production in Photon-Photon Collisions at $\sqrt{s}_{\mathrm{ee}}=161$ and $172 \mathrm{GeV}$
}

The OPAL Collaboration

\begin{abstract}
Di-jet production is studied in collisions of quasi-real photons radiated by the LEP beams at $\mathrm{e}^{+} \mathrm{e}^{-}$centre-of-mass energies $\sqrt{s}_{\mathrm{ee}}=161$ and $172 \mathrm{GeV}$. The jets are reconstructed using a cone jet finding algorithm. The angular distributions of direct and double-resolved processes are measured and compared to the predictions of leading order and next-to-leading order perturbative QCD. The jet energy profiles are also studied. The inclusive two-jet cross-section is measured as a function of $E_{\mathrm{T}}^{\mathrm{jet}}$ and $\left|\eta^{\mathrm{jet}}\right|$ and compared to next-to-leading order perturbative QCD calculations. The inclusive two-jet cross-section as a function of $\left|\eta^{\text {jet }}\right|$ is compared to the prediction of the leading order Monte Carlo generators PYTHIA and PHOJET. The Monte Carlo predictions are calculated with different parametrisations of the parton distributions of the photon. The influence of the 'underlying event' has been studied to reduce the model dependence of the predicted jet cross-sections from the Monte Carlo generators.
\end{abstract}

(To be submitted to European Physics Journal C) 


\section{The OPAL Collaboration}

G. Abbiendi ${ }^{2}$, K. Ackerstaff ${ }^{8}$, G. Alexander ${ }^{23}$, J. Allison ${ }^{16}$, N. Altekamp ${ }^{5}$, K.J. Anderson ${ }^{9}$, S. Anderson ${ }^{12}$, S. Arcelli ${ }^{17}$, S. Asai ${ }^{24}$, S.F. Ashby ${ }^{1}$, D. Axen ${ }^{29}$, G. Azuelos ${ }^{18, a}$, A.H. Ball ${ }^{17}$, E. Barberio ${ }^{8}$, T. Barillari ${ }^{2}$, R.J. Barlow ${ }^{16}$, R. Bartoldus ${ }^{3}$, J.R. Batley ${ }^{5}$, S. Baumann ${ }^{3}$, J. Bechtluft ${ }^{14}$, T. Behnke ${ }^{27}$, K.W. Bell ${ }^{20}$, G. Bella ${ }^{23}$, A. Bellerive ${ }^{9}$, S. Bentvelsen ${ }^{8}$, S. Bethke ${ }^{14}$, S. Betts ${ }^{15}$, O. Biebel ${ }^{14}$, A. Biguzzi ${ }^{5}$, S.D. Bird ${ }^{16}$, V. Blobel ${ }^{27}$, I.J. Bloodworth ${ }^{1}$, M. Bobinski ${ }^{10}$,

P. Bock ${ }^{11}$, J. Böhme ${ }^{14}$, D. Bonacorsi ${ }^{2}$, M. Boutemeur ${ }^{34}$, S. Braibant ${ }^{8}$, P. Bright-Thomas ${ }^{1}$,

L. Brigliadori ${ }^{2}$, R.M. Brown ${ }^{20}$, H.J. Burckhart ${ }^{8}$, C. Burgard ${ }^{8}$, R. Bürgin ${ }^{10}$, P. Capiluppi ${ }^{2}$, R.K. Carnegie ${ }^{6}$, A.A. Carter ${ }^{13}$, J.R. Carter $^{5}$, C.Y. Chang ${ }^{17}$, D.G. Charlton ${ }^{1, b}$, D. Chrisman ${ }^{4}$,

C. Ciocca ${ }^{2}$, P.E.L. Clarke ${ }^{15}$, E. Clay ${ }^{15}$, I. Cohen ${ }^{23}$, J.E. Conboy ${ }^{15}$, O.C. Cooke ${ }^{8}$, C. Couyoumtzelis ${ }^{13}$, R.L. Coxe ${ }^{9}$, M. Cuffiani ${ }^{2}$, S. Dado ${ }^{22}$, G.M. Dallavalle ${ }^{2}$, R. Davis ${ }^{30}$, S. De Jong $^{12}$, L.A. del Pozo ${ }^{4}$, A. de Roeck ${ }^{8}$, K. Desch ${ }^{8}$, B. Dienes ${ }^{33, d}$, M.S. Dixit ${ }^{7}$, J. Dubbert ${ }^{34}$, E. Duchovni ${ }^{26}$, G. Duckeck ${ }^{34}$, I.P. Duerdoth ${ }^{16}$, D. Eatough ${ }^{16}$, P.G. Estabrooks ${ }^{6}$, E. Etzion ${ }^{23}$,

H.G. Evans ${ }^{9}$, F. Fabbri² ${ }^{2}$, M.Fanti ${ }^{2}$, A.A.Faust ${ }^{30}$, F. Fiedler ${ }^{27}$, M. Fierro ${ }^{2}$, I. Fleck ${ }^{8}$, R. Folman ${ }^{26}$, A. Fürtjes ${ }^{8}$, D.I. Futyan ${ }^{16}$, P. Gagnon ${ }^{7}$, J.W. Gary ${ }^{4}$, J. Gascon ${ }^{18}$,

S.M. Gascon-Shotkin ${ }^{17}$, G. Gaycken ${ }^{27}$, C. Geich-Gimbel ${ }^{3}$, G. Giacomelli ${ }^{2}$, P. Giacomelli ${ }^{2}$, V. Gibson ${ }^{5}$, W.R. Gibson ${ }^{13}$, D.M. Gingrich ${ }^{30, a}$, D. Glenzinski ${ }^{9}$, J. Goldberg ${ }^{22}$, W. Gorn ${ }^{4}$,

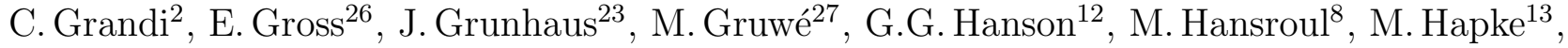

K. Harder ${ }^{27}$, C.K. Hargrove ${ }^{7}$, C. Hartmann ${ }^{3}$, M. Hauschild ${ }^{8}$, C.M. Hawkes ${ }^{5}$, R. Hawkings ${ }^{27}$,

R.J. Hemingway ${ }^{6}$, M.Herndon ${ }^{17}$, G. Herten ${ }^{10}$, R.D. Heuer ${ }^{8}$, M.D. Hildreth ${ }^{8}$, J.C. Hill ${ }^{5}$,

S.J.Hillier ${ }^{1}$, P.R. Hobson ${ }^{25}$, A. Hocker ${ }^{9}$, R.J.Homer ${ }^{1}$, A.K.Honma ${ }^{28, a}$, D. Horváth ${ }^{32, c}$,

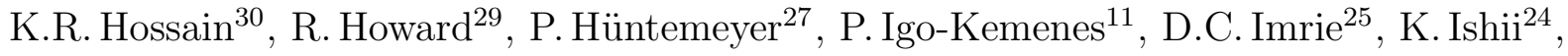

F.R. Jacob ${ }^{20}$, A. Jawahery ${ }^{17}$, H. Jeremie ${ }^{18}$, M. Jimack ${ }^{1}$, C.R. Jones ${ }^{5}$, P. Jovanovic ${ }^{1}$, T.R. Junk ${ }^{6}$,

D. Karlen ${ }^{6}$, V. Kartvelishvili ${ }^{16}$, K. Kawagoe ${ }^{24}$, T. Kawamoto ${ }^{24}$, P.I. Kayal ${ }^{30}$, R.K. Keeler ${ }^{28}$,

R.G. Kellogg ${ }^{17}$, B.W.Kennedy ${ }^{20}$, A. Klier ${ }^{26}$, S. Kluth ${ }^{8}$, T. Kobayashi ${ }^{24}$, M. Kobel ${ }^{3, e}$,

D.S. Koetke ${ }^{6}$, T.P. Kokott ${ }^{3}$, M. Kolrep ${ }^{10}$, S. Komamiya ${ }^{24}$, R.V.Kowalewski ${ }^{28}$, T. Kress ${ }^{11}$, P. Krieger ${ }^{6}$, J. von Krogh ${ }^{11}$, T. Kuhl ${ }^{3}$, P. Kyberd ${ }^{13}$, G.D. Lafferty ${ }^{16}$, D. Lanske ${ }^{14}$, J. Lauber ${ }^{15}$,

S.R. Lautenschlager ${ }^{31}$, I. Lawson ${ }^{28}$, J.G. Layter ${ }^{4}$, D. Lazic ${ }^{22}$, A.M. Lee ${ }^{31}$, D. Lellouch ${ }^{26}$, J. Letts ${ }^{12}$, L. Levinson ${ }^{26}$, R. Liebisch ${ }^{11}$, B. List ${ }^{8}$, C. Littlewood ${ }^{5}$, A.W. Lloyd ${ }^{1}$, S.L. Lloyd ${ }^{13}$,

F.K. Loebinger ${ }^{16}$, G.D. Long ${ }^{28}$, M.J. Losty ${ }^{7}$, J. Ludwig ${ }^{10}$, D. Liu ${ }^{12}$, A. Macchiolo ${ }^{2}$,

A. Macpherson ${ }^{30}$, W. Mader ${ }^{3}$, M. Mannelli ${ }^{8}$, S. Marcellini ${ }^{2}$, C. Markopoulos ${ }^{13}$, A.J. Martin ${ }^{13}$, J.P. Martin ${ }^{18}$, G. Martinez ${ }^{17}$, T. Mashimo ${ }^{24}$, P. Mättig ${ }^{26}$, W.J. McDonald ${ }^{30}$, J. McKenna ${ }^{29}$, E.A. Mckigney ${ }^{15}$, T.J. McMahon ${ }^{1}$, R.A. McPherson ${ }^{28}$, F. Meijers ${ }^{8}$, S. Menke ${ }^{3}$, F.S. Merritt ${ }^{9}$,

H. Mes ${ }^{7}$, J. Meyer ${ }^{27}$, A. Michelini' ${ }^{2}$, S. Mihara ${ }^{24}$, G. Mikenberg ${ }^{26}$, D.J. Miller ${ }^{15}$, R. Mir ${ }^{26}$, W. Mohr ${ }^{10}$, A. Montanari ${ }^{2}$, T. Mori ${ }^{24}$, K. Nagai ${ }^{8}$, I. Nakamura ${ }^{24}$, H.A. Neal ${ }^{12}$, B. Nellen ${ }^{3}$, R. Nisius ${ }^{8}$, S.W. O’Neale ${ }^{1}$, F.G. Oakham ${ }^{7}$, F. Odorici ${ }^{2}$, H.O. Ogren ${ }^{12}$, M.J. Oreglia ${ }^{9}$, S. Orito ${ }^{24}$, J. Pálinkás ${ }^{33, d}$, G. Pásztor ${ }^{32}$, J.R. Pater ${ }^{16}$, G.N.Patrick ${ }^{20}$, J. Patt ${ }^{10}$, R. Perez-Ochoa ${ }^{8}$, S. Petzold ${ }^{27}$, P. Pfeifenschneider ${ }^{14}$, J.E. Pilcher ${ }^{9}$, J. Pinfold ${ }^{30}$, D.E. Plane ${ }^{8}$, P. Poffenberger ${ }^{28}$, J. Polok ${ }^{8}$, M. Przybycień ${ }^{8}$, C. Rembser ${ }^{8}$, H. Rick ${ }^{8}$, S. Robertson ${ }^{28}$, S.A. Robins ${ }^{22}$, N. Rodning ${ }^{30}$, J.M. Roney ${ }^{28}$, K. Roscoe ${ }^{16}$, A.M. Rossi ${ }^{2}$, Y.Rozen ${ }^{22}$, K. Runge ${ }^{10}$, O. Runolfsson ${ }^{8}$, D.R. Rust ${ }^{12}$, K. Sachs ${ }^{10}$, T.Saeki ${ }^{24}$, O.Sahr ${ }^{34}$, W.M.Sang ${ }^{25}$, E.K.G.Sarkisyan ${ }^{23}$, C.Sbarra ${ }^{29}$, A.D.Schaile ${ }^{34}$,

O. Schaile ${ }^{34}$, F. Scharf ${ }^{3}$, P.Scharff-Hansen ${ }^{8}$, J. Schieck ${ }^{11}$, B. Schmitt ${ }^{8}$, S. Schmitt ${ }^{11}$,

A. Schöning ${ }^{8}$, M. Schröder ${ }^{8}$, M. Schumacher ${ }^{3}$, C.Schwick ${ }^{8}$, W.G. Scott ${ }^{20}$, R. Seuster ${ }^{14}$, T.G. Shears ${ }^{8}$, B.C. Shen ${ }^{4}$, C.H. Shepherd-Themistocleous ${ }^{8}$, P. Sherwood ${ }^{15}$, G.P. Siroli ${ }^{2}$, A. Sittler ${ }^{27}$, A.Skuja ${ }^{17}$, A.M. Smith ${ }^{8}$, G.A.Snow ${ }^{17}$, R. Sobie ${ }^{28}$, S. Söldner-Rembold ${ }^{10}$, M.Sproston ${ }^{20}$, A. Stahl ${ }^{3}$, K. Stephens ${ }^{16}$, J.Steuerer ${ }^{27}$, K. Stoll ${ }^{10}$, D. Strom ${ }^{19}$, R. Ströhmer ${ }^{34}$, 


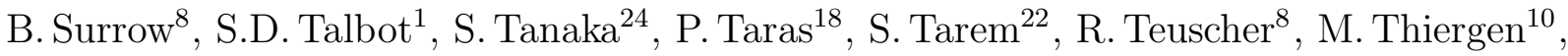
M.A. Thomson ${ }^{8}$, E. von Törne ${ }^{3}$, E. Torrence ${ }^{8}$, S. Towers ${ }^{6}$, I. Trigger ${ }^{18}$, Z. Trócsányi ${ }^{33}$, E. Tsur ${ }^{23}$, A.S. Turcot ${ }^{9}$, M.F. Turner-Watson ${ }^{8}$, R. Van Kooten ${ }^{12}$, P. Vannerem ${ }^{10}$, M. Verzocchi ${ }^{10}$, H. Voss $^{3}$, F. Wäckerle ${ }^{10}$, A. Wagner ${ }^{27}$, C.P. Ward ${ }^{5}$, D.R. Ward ${ }^{5}$, P.M. Watkins ${ }^{1}$, A.T. Watson ${ }^{1}$, N.K. Watson ${ }^{1}$, P.S. Wells ${ }^{8}$, N. Wermes ${ }^{3}$, J.S. White ${ }^{6}$, G.W.Wilson ${ }^{16}$, J.A. Wilson ${ }^{1}$, T.R. Wyatt ${ }^{16}$, S. Yamashita ${ }^{24}$, G. Yekutieli ${ }^{26}$, V. Zacek ${ }^{18}$, D. Zer-Zion ${ }^{8}$

${ }^{1}$ School of Physics and Astronomy, University of Birmingham, Birmingham B15 2TT, UK ${ }^{2}$ Dipartimento di Fisica dell' Università di Bologna and INFN, I-40126 Bologna, Italy

${ }^{3}$ Physikalisches Institut, Universität Bonn, D-53115 Bonn, Germany

${ }^{4}$ Department of Physics, University of California, Riverside CA 92521, USA

${ }^{5}$ Cavendish Laboratory, Cambridge CB3 0HE, UK

${ }^{6}$ Ottawa-Carleton Institute for Physics, Department of Physics, Carleton University, Ottawa, Ontario K1S 5B6, Canada

${ }^{7}$ Centre for Research in Particle Physics, Carleton University, Ottawa, Ontario K1S 5B6, Canada

${ }^{8}$ CERN, European Organisation for Particle Physics, CH-1211 Geneva 23, Switzerland

${ }^{9}$ Enrico Fermi Institute and Department of Physics, University of Chicago, Chicago IL 60637, USA

${ }^{10}$ Fakultät für Physik, Albert-Ludwigs-Universität, D-79104 Freiburg, Germany

${ }^{11}$ Physikalisches Institut, Universität Heidelberg, D-69120 Heidelberg, Germany

${ }^{12}$ Indiana University, Department of Physics, Swain Hall West 117, Bloomington IN 47405, USA

${ }^{13}$ Queen Mary and Westfield College, University of London, London E1 4NS, UK

${ }^{14}$ Technische Hochschule Aachen, III Physikalisches Institut, Sommerfeldstrasse 26-28, D-52056

Aachen, Germany

${ }^{15}$ University College London, London WC1E 6BT, UK

${ }^{16}$ Department of Physics, Schuster Laboratory, The University, Manchester M13 9PL, UK

${ }^{17}$ Department of Physics, University of Maryland, College Park, MD 20742, USA

${ }^{18}$ Laboratoire de Physique Nucléaire, Université de Montréal, Montréal, Quebec H3C 3J7, Canada

${ }^{19}$ University of Oregon, Department of Physics, Eugene OR 97403, USA

${ }^{20}$ CLRC Rutherford Appleton Laboratory, Chilton, Didcot, Oxfordshire OX11 0QX, UK

${ }^{22}$ Department of Physics, Technion-Israel Institute of Technology, Haifa 32000, Israel

${ }^{23}$ Department of Physics and Astronomy, Tel Aviv University, Tel Aviv 69978, Israel

${ }^{24}$ International Centre for Elementary Particle Physics and Department of Physics, University of Tokyo, Tokyo 113, and Kobe University, Kobe 657, Japan

${ }^{25}$ Institute of Physical and Environmental Sciences, Brunel University, Uxbridge, Middlesex UB8 3PH, UK

${ }^{26}$ Particle Physics Department, Weizmann Institute of Science, Rehovot 76100, Israel

${ }^{27}$ Universität Hamburg/DESY, II Institut für Experimental Physik, Notkestrasse 85, D-22607 Hamburg, Germany

${ }^{28}$ University of Victoria, Department of Physics, P O Box 3055, Victoria BC V8W 3P6, Canada ${ }^{29}$ University of British Columbia, Department of Physics, Vancouver BC V6T 1Z1, Canada

${ }^{30}$ University of Alberta, Department of Physics, Edmonton AB T6G 2J1, Canada

${ }^{31}$ Duke University, Dept of Physics, Durham, NC 27708-0305, USA 
${ }^{32}$ Research Institute for Particle and Nuclear Physics, H-1525 Budapest, P O Box 49, Hungary

${ }^{33}$ Institute of Nuclear Research, H-4001 Debrecen, P O Box 51, Hungary

${ }^{34}$ Ludwigs-Maximilians-Universität München, Sektion Physik, Am Coulombwall 1, D-85748 Garching, Germany

${ }^{a}$ and at TRIUMF, Vancouver, Canada V6T 2A3

${ }^{b}$ and Royal Society University Research Fellow

${ }^{c}$ and Institute of Nuclear Research, Debrecen, Hungary

${ }^{d}$ and Department of Experimental Physics, Lajos Kossuth University, Debrecen, Hungary

${ }^{e}$ on leave of absence from the University of Freiburg

\section{Introduction}

We present a study of di-jet production in photon-photon collisions at $\sqrt{s}_{\mathrm{ee}}=161$ and $172 \mathrm{GeV}$ with an integrated luminosity of $20 \mathrm{pb}^{-1}$. The cone jet finding algorithm was used to reconstruct jets. The production of di-jet events in the collision of two quasi-real photons can be used to study the structure of the photon and to test QCD predictions. At $\mathrm{e}^{+} \mathrm{e}^{-}$colliders the photons are emitted by the beam electrons]. Most of the photons carry only a small squared fourmomentum, $Q^{2}$, and can be considered to be quasi-real $\left(Q^{2} \approx 0\right)$. Accordingly, the electrons are scattered with very small angles and are not detected. Events where one or both scattered electrons are detected are vetoed ("anti-tagged").

The interactions of the photons can be modelled by assuming that each photon can either interact directly or appear resolved through its fluctuations into hadronic components. The interaction of two photons can be classified either as a direct process where two bare photons interact, a single-resolved process where a bare photon interacts with a parton (quark or gluon) of the other photon or a double-resolved process where partons of both photons interact. The possibility to distinguish between direct and resolved processes in di-jet events has already been demonstrated by OPAL at $\sqrt{s}_{\text {ee }}=130-136 \mathrm{GeV}$ [1]. Depending on the type of photon-photon interaction different matrix elements for the QCD scattering process contribute. These matrix elements have been calculated in leading order (LO) [2] and next-to-leading order (NLO) [3]. The matrix element of the scattering between two bare photons is the one for the process $\gamma \gamma \rightarrow q \bar{q}$. In double-resolved processes the matrix elements of quark-quark, gluon-quark and gluon-gluon scattering are involved [2]. These calculations predict different distributions of the parton scattering angle in the centre-of-mass system of the colliding particles. In hadron-hadron and photon-hadron interactions similar QCD predictions have already been confirmed [4, 5].

The investigation of the internal structure of jets gives insight into the transition between a parton produced in a hard process and the observable hadrons which originate from the fragmentation process [6]. The dependence of the jet shapes on QCD parton radiation calculated in the Leading-Log Approximation (LLA), and the differences between the jet shapes of direct and resolved processes, have recently been measured at HERA [7].

\footnotetext{
${ }^{1}$ Positrons are also referred to as electrons
} 
The measurement of inclusive jet cross-sections in $\gamma \gamma$ and $\gamma p$ interactions can constrain the gluonic content of the photon [8, 9]. This is done by comparing the jet cross-sections to the LO QCD models PYTHIA and PHOJET, using different parametrisations of the parton distribution functions of the photon, and to NLO QCD calculations [10, 11]. PYTHIA and PHOJET also model the so-called 'underlying event' by multiple interactions involving partons from the remnants of the same two initial photons, whereas the NLO QCD calculations do not take into account such effects. In the models, the contribution from multiple interactions to the jet cross-sections has to be tuned to increase sensitivity to the parton distributions of the photon. In contrast to deep inelastic electron-photon scattering [12], which in leading order is only sensitive to the quark content of the photon, the gluon content of the photon can be tested directly in the resolved interaction of two almost real photons, where the fraction of gluon initiated processes is large.

Inclusive one-jet and two-jet cross-sections in photon-photon collisions have previously been measured at an $\mathrm{e}^{+} \mathrm{e}^{-}$centre-of-mass energy of $\sqrt{s}_{\mathrm{ee}}=58 \mathrm{GeV}$ at TRISTAN 8, 13] and at an $\mathrm{e}^{+} \mathrm{e}^{-}$centre-of-mass energy of $\sqrt{s}_{\mathrm{ee}}=130$ and $136 \mathrm{GeV}$ at LEP [1]. This paper extends our analysis at lower energies in which a similar strategy was used. Jets are studied in a wider kinematic range and with higher integrated luminosity. In addition, we present new results on angular distributions, jet shapes and energy flows.

\section{Process kinematics}

The properties of the interacting photons are described by their negative squared four-momentum transfers, $Q_{i}^{2}$. Each $Q_{i}^{2}$ is related to the electron scattering angle $\Theta_{i}^{\prime}$ relative to the beam direction by

$$
Q_{i}^{2}=-\left(p_{i}-p_{i}^{\prime}\right)^{2} \approx 2 E_{i} E_{i}^{\prime}\left(1-\cos \Theta_{i}^{\prime}\right),
$$

where $p_{i}$ and $p_{i}^{\prime}$ are the four-momenta of the beam electrons and the scattered electrons, respectively, and $E_{i}$ and $E_{i}^{\prime}$ are their energies. Events with detected scattered electrons (single-tagged or double-tagged events) are excluded from the analysis. This anti-tagging condition defines an upper limit on $Q_{i}^{2}$ for both photons. This condition is met when the scattering angle $\Theta^{\prime}$ of the electrons is less than $\Theta_{\max }^{\prime}$, where $\Theta_{\max }^{\prime}$ is the angle between the beam-axis and the inner edge of the detector. The squared invariant mass of the hadronic final state

$$
W^{2}=\left(\sum_{h} E_{h}\right)^{2}-\left(\sum_{h} \vec{p}_{h}\right)^{2}
$$

is calculated by summing over the energies, $E_{h}$, and momenta, $\vec{p}_{h}$, of all final state hadrons. The spectrum of photons with an energy fraction $y$ of the electron beam may be obtained by the Equivalent Photon Approximation (EPA) [14]:

$$
f_{\gamma / \mathrm{e}}(y)=\frac{\alpha}{2 \pi}\left(\frac{1+(1-y)^{2}}{y} \log \frac{Q_{\max }^{2}}{Q_{\min }^{2}}-2 m_{\mathrm{e}}^{2} y\left(\frac{1}{Q_{\min }^{2}}-\frac{1}{Q_{\max }^{2}}\right)\right),
$$

with $\alpha$ being the electromagnetic coupling constant. The minimum kinematically allowed squared four-momentum transfer, $Q_{\min }^{2}$, is determined by the electron mass $m_{\mathrm{e}}$ :

$$
Q_{\min }^{2}=\frac{m_{\mathrm{e}}^{2} y^{2}}{1-y} \text {. }
$$


The effective maximum four-momentum transfer $Q_{\max }^{2}$ is given by the anti-tagging condition, i.e. the requirement that both electrons remain undetected.

\section{The OPAL detector}

A detailed description of the OPAL detector can be found in Ref. [15], and therefore only a brief account of the main features relevant to the present analysis will be given here.

The central tracking system is located inside a solenoidal magnet which provides a uniform axial magnetic field of $0.435 \mathrm{~T}$ along the beam axist 7 . The magnet is surrounded in the barrel region $(|\cos \theta|<0.82)$ by a lead glass electromagnetic calorimeter (ECAL) and a hadronic sampling calorimeter (HCAL). Outside the HCAL, the detector is surrounded by muon chambers. There are similar layers of detectors in the endcaps $(0.82<|\cos \theta|<0.98)$. The small angle region from 47 to $140 \mathrm{mrad}$ around the beam pipe on both sides of the interaction point is covered by the forward calorimeters (FD) and the region from 25 to $59 \mathrm{mrad}$ by the silicon tungsten luminometers (SW) [16]. From 1996 onwards, relevant to the data presented in this paper, the lower boundary of the acceptance has been increased to $33 \mathrm{mrad}$ following the installation of a low angle shield to protect the central tracking system against possible synchrotron radiation.

Starting with the innermost components, the tracking system consists of a high precision silicon microvertex detector, a vertex drift chamber, a large volume jet chamber with 159 layers of axial anode wires and a set of $z$ chambers measuring the track coordinates along the beam direction. The transverse momenta, $p_{\mathrm{T}}$, of tracks are measured with a precision

parametrised by $\sigma_{p_{\mathrm{T}}} / p_{\mathrm{T}}=\sqrt{0.02^{2}+\left(0.0015 \cdot p_{\mathrm{T}}\right)^{2}}\left(p_{\mathrm{T}}\right.$ in $\left.\mathrm{GeV} / c\right)$ in the central region. In this paper "transverse" is always defined with respect to the $z$ axis. The jet chamber also provides measurements of the energy loss, $\mathrm{d} E / \mathrm{d} x$, which are used for particle identification [15].

The barrel and endcap sections of the ECAL are both constructed from lead glass blocks with a depth of 24.6 radiation lengths in the barrel region and more than 22 radiation lengths in the endcaps. The FD consist of cylindrical lead-scintillator calorimeters with a depth of 24 radiation lengths divided azimuthally into 16 segments. The electromagnetic energy resolution is about $18 \% / \sqrt{E}$, where $E$ is in $\mathrm{GeV}$. The SW detectors consist of 19 layers of silicon detectors and 18 layers of tungsten, corresponding to a total of 22 radiation lengths. Each silicon layer consists of 16 wedge shaped silicon detectors. The electromagnetic energy resolution is about $25 \% / \sqrt{E}(E$ in $\mathrm{GeV})$.

\section{Event selection and jet finding}

Two-photon events are selected with the following set of cuts:

- The sum of all energy deposits in the ECAL and the HCAL has to be less than $45 \mathrm{GeV}$.

\footnotetext{
${ }^{2}$ In the OPAL coordinate system the $z$ axis points in the direction of the $\mathrm{e}^{-}$beam. The polar angle $\theta$, the azimuthal angle $\phi$ and the radius $r$ denote the usual spherical coordinates.
} 
Calorimeter clusters have to pass an energy threshold of $100 \mathrm{MeV}$ in the barrel section or $250 \mathrm{MeV}$ in the endcap section for the ECAL and of $600 \mathrm{MeV}$ for the barrel and endcap section of the HCAL.

- The visible invariant mass measured in the ECAL has to be greater than $3 \mathrm{GeV}$.

- The missing transverse energy of the event measured in the ECAL and the FD has to be less than $5 \mathrm{GeV}$. For a FD cluster to be counted its energy has to be larger than $1 \mathrm{GeV}$.

- At least 5 tracks must have been found in the tracking chambers. A track is required to have a minimum transverse momentum of $120 \mathrm{MeV} / c$, at least 20 hits in the central jet chamber, and the innermost hit of the track must be within a radius of $60 \mathrm{~cm}$ with respect to the $z$ axis. The distance of the point of closest approach to the origin in the $r \phi$ plane must be less than $30 \mathrm{~cm}$ in the $z$ direction and less than $2 \mathrm{~cm}$ in the $r \phi$ plane. Tracks with a momentum error larger than the momentum itself are rejected if they have fewer than 80 hits. The number of measured hits in the jet chamber must be more than half of the number of possible hits, where the number of possible hits is calculated from the polar angle $\theta$ of the track, assuming that the track has no curvature.

- To remove events with scattered electrons in the FD or in the SW calorimeters, the total energy sum measured in the FD has to be less than $50 \mathrm{GeV}$ and the total energy sum measured in the SW calorimeter has to be less than $35 \mathrm{GeV}$. A cluster in the SW calorimeter is accepted if it has an energy of more than $1 \mathrm{GeV}$. These cuts also reduce the contamination from multihadronic annihilation events with their thrust axis close to the beam direction.

- In order to estimate the $z$ position of the primary vertex, we calculate the error-weighted average $\left\langle z_{0}\right\rangle$ of the $z$ coordinates of all tracks at the point of closest approach to the origin in the $r \phi$ plane. The background due to beam-gas interactions is reduced by requiring $\left|\left\langle z_{0}\right\rangle\right|<10 \mathrm{~cm}$ and $|Q| \leq 3$, where $Q$ is the net charge of an event calculated from adding the charges of all tracks.

- To remove beam-wall events the radial distance of the primary vertex from the beam axis has to be less than $3 \mathrm{~cm}$.

In the cone jet finding algorithm the total transverse energy $E_{\mathrm{T}}^{\mathrm{jet}}$ of the jet inside the cone is the scalar sum of the transverse energies of its components [1, 17]. In all parts of this analysis, a sum over the particles in the event or in a jet means a sum over tracks satisfying the above quality cuts, and over all calorimeters clusters, including the FD and SW calorimeters. An algorithm is applied to avoid double-counting of particle momenta in the central tracking system and their energy deposits in the calorimeters [1]. The transverse energy $E_{\mathrm{T}_{i}}$ of a particle $i$ is defined relative to the $z$ axis of the detector with $E_{\mathrm{T}_{i}}=E_{i} \sin \theta_{i}$. For a cone jet to be accepted, the value of $E_{\mathrm{T}}^{\text {jet }}$ must be greater than a certain minimum $E_{\mathrm{T}}^{\mathrm{min}}$. The results of the cone jet finding algorithm depend on $E_{\mathrm{T}}^{\mathrm{min}}$ and the cone size $R=\sqrt{(\Delta \eta)^{2}+(\Delta \phi)^{2}}$ with pseudorapidity $\eta=-\ln \tan (\theta / 2)$ and azimuthal angle $\phi$. Here the values were chosen to be $R=1$ and $E_{\mathrm{T}}^{\min }=2 \mathrm{GeV}$. The jet pseudorapidity in the laboratory frame is required to be within $\left|\eta^{\text {jet }}\right|<2$. Monte Carlo studies have shown that jets with $\left|\eta^{\text {jet }}\right|<2$ are well reconstructed and are normally fully contained in the detector. This leads to a wider $\eta^{\text {jet }}$ acceptance than in our previous analysis [1]. 
We use data corresponding to an integrated luminosity of $9.9 \mathrm{pb}^{-1}$ at $\sqrt{s}_{\mathrm{ee}}=161 \mathrm{GeV}$ and $10.0 \mathrm{pb}^{-1}$ at $\sqrt{s}_{\text {ee }}=172 \mathrm{GeV}$. After applying all cuts and requiring at least two jets with $E_{\mathrm{T}}^{\text {jet }}>3 \mathrm{GeV}$ and $\left|\eta^{\text {jet }}\right|<2,2845$ events remain, equally divided between the two centreof-mass energies. For the purpose of this analysis, the difference between the data taken at $\sqrt{s}_{\text {ee }}=161 \mathrm{GeV}$ and at $172 \mathrm{GeV}$ is small and therefore the distributions for both energies have been added. About 12.3\% of the di-jet events contain 3 or more jets. In events with more than two jets, only the two jets with the highest $E_{\mathrm{T}}^{\text {jet }}$ values are taken.

\section{Monte Carlo simulation}

The Monte Carlo generators PYTHIA 5.722 [18, 19] and PHOJET 1.05c [20] are used, both based on LO QCD calculations. These generators have been optimised to describe $\gamma \mathrm{p}$ and $\overline{\mathrm{p}} \mathrm{p}$ interactions. The probability of finding a parton in the photon is taken from parametrisations of the parton distribution functions. The SaS-1D parametrisation [21] is used as default in PYTHIA and the LO GRV parametrisation [22] as default in PHOJET. All possible hard interactions of quarks, gluons and photons are simulated using LO matrix elements for massless quarks. The default value of the cutoff on the transverse momentum of the two outgoing partons of $\hat{p}_{\mathrm{t}}^{\min }=1.4 \mathrm{GeV} / c$ is used. More details can be found in Ref. [1].

The incoming photons in double-resolved events can be viewed as beams of partons. For small parton transverse momenta the LO parton scattering cross-section diverges and becomes larger than the non-diffractive cross-section as measured in $\gamma p$ collisions. If more than one parton scattering process is allowed in one event the problem of too large parton cross-section can be solved. These multiple interactions are calculated as LO QCD processes between partons of the photon remnants. In PYTHIA a lower cutoff parameter $p_{\mathrm{t}}^{\mathrm{mi}}$ is introduced, which describes the transverse momentum of the parton and is set by default to $1.4 \mathrm{GeV} / c$. The fluctuations of the number of hard interactions are calculated from a Poisson distribution. The average number $n_{\mathrm{mi}}$ of interactions in double-resolved di-jet events simulated by PYTHIA is 1.3 for the default setting $p_{\mathrm{t}}^{\mathrm{mi}}=1.4 \mathrm{GeV} / c$ using SaS-1D. PYTHIA and PHOJET use multiple interactions as a component to model the underlying event.

In PHOJET the $Q^{2}$ suppression of the total $\gamma \gamma$ cross-section is parametrised using Generalised Vector Meson Dominance (GVMD). A model for the change of soft hadron production and diffraction with increasing photon virtuality $Q^{2}$ is also included. The photon-photon mode of PYTHIA only simulates the interactions of real photons with $Q^{2}=0$. The virtuality of the photons defined by $Q^{2}$ enters only through the Equivalent Photon Approximation in the generation of the photon energy spectrum, but the electrons are scattered at zero angle. This model is not expected to be correct for larger values of $Q^{2}$. The contribution of di-jet events with $Q^{2}>1 \mathrm{GeV}^{2}$ and $\Theta^{\prime}<33 \mathrm{mrad}$ generated with the electron-photon mode of PYTHIA is small and therefore neglected.

The fragmentation of the parton final state is handled in both generators by the routines of JETSET 7.408 [18]. Initial and final state parton radiation is included based on the LLA.

All signal and background Monte Carlo samples apart from beam-gas and beam-wall events were generated with full simulation of the OPAL detector [23. They are analysed using the 
same reconstruction algorithms as are applied to the data.

The median $Q^{2}$ of the selected PHOJET events is of the order $10^{-4} \mathrm{GeV}^{2}$. The visible hadronic invariant mass, $W_{\text {vis }}$, measured with all detector information is well described within the errors of the measurements by the Monte Carlo simulations. A detailed comparison between $W_{\text {vis }}$ and the generated $W$ can be found in Ref. [24].

After applying the detector simulation and the selection cuts to these events, about $83 \%$ of all generated Monte Carlo events with at least two hadron jets in the range $E_{\mathrm{T}}^{\mathrm{jet}}>3 \mathrm{GeV}$ and $\left|\eta^{\text {jet }}\right|<2$ are selected. The trigger efficiency for all selected Monte Carlo events with at least two reconstructed jets in the detector is close to $100 \%$. The number of background events is small, about $1.5 \%$ in total. About $1.1 \%$ of the events in the data sample are expected to be $\mathrm{e}^{+} \mathrm{e}^{-}$annihilation events with hadronic final states and $0.4 \% \mathrm{e}^{+} \mathrm{e}^{-} \rightarrow \mathrm{e}^{+} \mathrm{e}^{-} \tau^{+} \tau^{-}$events. No significant background from beam-gas or beam-wall events is observed.

\section{$6 \quad$ Properties of direct and resolved processes}

In LO QCD, neglecting multiple parton interactions, two hard parton jets are produced in $\gamma \gamma$ interactions. In single- or double-resolved interactions, the two hard parton jets are expected to be accompanied by one or two remnant jets.

A pair of variables, $x_{\gamma}^{+}$and $x_{\gamma}^{-}$, can be defined [25] which specify the fraction of the photon's momentum participating in the hard scattering:

$$
x_{\gamma}^{+}=\frac{\sum_{\text {jets }=1,2}\left(E+p_{z}\right)}{\sum_{\text {hadrons }}\left(E+p_{z}\right)} \text { and } x_{\gamma}^{-}=\frac{\sum_{\text {jets }=1,2}\left(E-p_{z}\right)}{\sum_{\text {hadrons }}\left(E-p_{z}\right)},
$$

where $p_{z}$ is the momentum component along the $z$ axis of the detector and $E$ is the energy of the jets or hadrons. These variables give some separation between direct and resolved di-jet events [1].

Ideally, for direct events without remnant jets, the total energy of the event is contained in the two jets, i.e. $x_{\gamma}^{+}=1$ and $x_{\gamma}^{-}=1$, whereas for single-resolved events either $x_{\gamma}^{+}$or $x_{\gamma}^{-}$and for double-resolved events both values, $x_{\gamma}^{+}$and $x_{\gamma}^{-}$, are expected to be smaller than 1. Samples with large direct and double-resolved contributions can be separated by requiring both $x_{\gamma}^{+}$and $x_{\gamma}^{-}$to be larger than 0.8 (denoted as $x_{\gamma}^{ \pm}>0.8$ ) or both values to be smaller than 0.8 (denoted as $\left.x_{\gamma}^{ \pm}<0.8\right)$, respectively. Details about the separation between different event classes can be found in Ref. [1]. In the PYTHIA Monte Carlo using the SaS-1D parametrisation $86 \%$ of all events in the region $x_{\gamma}^{ \pm}>0.8$ originate from direct interactions and $81 \%$ of all events in the region $x_{\gamma}^{ \pm}<0.8$ originate from double-resolved interactions.

The $x_{\gamma}$ distribution is shown in Figure 1 in bins of $\bar{E}_{\mathrm{T}}^{\mathrm{jet}}$, where

$$
\bar{E}_{\mathrm{T}}^{\mathrm{jet}}=\frac{E_{\mathrm{T}}^{\mathrm{jet} 1}+E_{\mathrm{T}}^{\mathrm{jet} 2}}{2}
$$


is the mean value of the transverse energies $E_{\mathrm{T}}^{\text {jet1 }}$ and $E_{\mathrm{T}}^{\text {jet2 }}$ of the two jets. Each event is added to the plot twice, at the values of $x_{\gamma}^{+}$and of $x_{\gamma}^{-}$. No correction for selection cuts and detector effects has been applied, but the background has been subtracted using the Monte Carlo. The Monte Carlo predictions of PYTHIA and PHOJET are normalised to the number of events observed in the data. The contribution from direct processes, as predicted from PYTHIA, is also shown. The events from direct processes are concentrated at high $x_{\gamma}$ values. In Fig. 1 1 a, at low $\bar{E}_{\mathrm{T}}^{\text {jet }}$, the direct part contributes to about $17 \%$ to the total number of events. As $\bar{E}_{\mathrm{T}}^{\text {jet }}$ increases, the $x_{\gamma}$ distribution shifts to higher values and the fraction of direct events in the PYTHIA sample increases to $68 \%$ for $12<\bar{E}_{\mathrm{T}}^{\text {jet }}<20 \mathrm{GeV}$ (Fig. 11d). The number of events is underestimated by PYTHIA and PHOJET by about $25-30 \%$, if the predicted Monte Carlo cross-sections are taken into account, mainly for $x_{\gamma}<0.9$.

\section{Angular distributions in direct and resolved events}

Since the jets in double-resolved events do not contain all of the hadronic activity it is expected that there will be more energy flow outside the jets in double-resolved events than in direct events. Fig. 2 shows the energy flow transverse to the beam direction as a function of $\Delta \eta^{\prime}$ measured with respect to the jet direction for data samples with different $x_{\gamma}^{ \pm}$cuts. The pseudorapidity difference is defined by:

$$
\Delta \eta^{\prime}=k\left(\eta-\eta^{\mathrm{jet}}\right)
$$

where $\eta$ is the pseudorapidity of the cluster or the track. The factor $k$ is chosen event-byevent to be $k=+1$ for events with $x_{\gamma}^{+}>x_{\gamma}^{-}$and $k=-1$ for events with $x_{\gamma}^{+}<x_{\gamma}^{-}$. As a consequence, there is always more of the remnant found at $\Delta \eta^{\prime}<0$ and the enhancement due to the additional transverse energy flow observed at negative and positive $\Delta \eta^{\prime}$ is asymmetric. No correction for acceptance or resolution effects has been applied. The energy flow is integrated over $|\Delta \phi|<\pi / 2$. The jets in the data sample with $x_{\gamma}^{ \pm}>0.8$ (Fig. 2a) are more collimated and there is almost no activity outside the jet, whereas the transverse energy flow of two-jet events with $x_{\gamma}^{ \pm}<0.8$ (Fig. 2 $\mathrm{b}$ ) shows considerable activity outside the jets for $\left|\Delta \eta^{\prime}\right|>1$. The energy flow outside the jets is well modelled by PYTHIA whereas PHOJET shows a wider distribution.

In the di-jet centre-of-mass frame one expects different angular distributions for direct and double-resolved events. An estimator of the angle $\theta^{*}$ between the jet axis and the axis of the incoming partons or direct photons in the di-jet centre-of-mass frame can be formed from the jet pseudorapidities:

$$
\cos \theta^{*}=\tanh \left(\frac{\eta^{\mathrm{jet} 1}-\eta^{\mathrm{jet} 2}}{2}\right) .
$$

The two jets are assumed to be collinear in $\phi$ with $E_{\mathrm{T}}^{\text {jet1 }}=E_{\mathrm{T}}^{\text {jet2 }}$. Since the ordering of the jets in the detector is arbitrary, only $\left|\cos \theta^{*}\right| \operatorname{can}$ be measured. The matrix elements of elastic partonparton scattering processes are known to LO [2]. For a given parton centre-of-mass energy the cross-sections vary only with the scattering angle $\theta^{*}$. The LO direct process $\gamma \gamma \rightarrow \mathrm{q} \overline{\mathrm{q}}$ is mediated by $t$-channel spin- $\frac{1}{2}$ quark exchange which leads to an angular dependence $\propto(1-$ $\left.\cos ^{2} \theta^{*}\right)^{-1}$. In double-resolved processes all matrix elements involving quarks and gluons have to be taken into account, with a large contribution from spin-0 gluon exchange. After adding 
up all relevant processes, perturbative QCD predicts an angular dependence of approximately $\propto\left(1-\left|\cos \theta^{*}\right|\right)^{-2}[2]$.

In order to measure the $\cos \theta^{*}$ distribution, additional cuts have to be applied. These cuts minimise kinematic biases and improve the detector resolution on $\cos \theta^{*}$. The invariant mass of the two-jet system is calculated as

$$
M_{\mathrm{jj}} \approx \frac{2 E_{\mathrm{T}}^{\mathrm{jet}}}{\sqrt{1-\left|\cos \theta^{*}\right|^{2}}} .
$$

The cut on $E_{\mathrm{T}}^{\mathrm{jet}}>3 \mathrm{GeV}$ restricts the accessible range of values of $\left|\cos \theta^{*}\right|$. For $M_{\mathrm{jj}}<10 \mathrm{GeV}$ the number of events decreases because of the $E_{\mathrm{T}}^{\text {jet }}$ cut. Requiring $M_{\mathrm{jj}}$ to be larger than $12 \mathrm{GeV}$ ensures that the distribution in the range $\left|\cos \theta^{*}\right|<0.85$ is not biased by the $E_{\mathrm{T}}^{\mathrm{jet}}$ cut. The Lorentz boost of the two-jet system in the $z$ direction is defined by

$$
\bar{\eta}=\frac{\eta^{\mathrm{jet} 1}+\eta^{\mathrm{jet} 2}}{2}
$$

since in the two-jet centre-of-mass system $\bar{\eta}^{*}=0$. Events with $|\bar{\eta}|>1$ were rejected because the detector resolution on $\left|\cos \theta^{*}\right|$ deteriorates significantly for events with $|\bar{\eta}|>1$. After additionally requiring $M_{\mathrm{jj}}>12 \mathrm{GeV}$ and $|\bar{\eta}|<1150$ data events remain with $x_{\gamma}^{ \pm}>0.8$ and 350 data events with $x_{\gamma}^{ \pm}<0.8$.

Table 1 shows the measured cross-sections for data samples with large direct and large double-resolved contributions according to the separation with $x_{\gamma}^{+}$and $x_{\gamma}^{-}$. The cross-section in each bin of $\left|\cos \theta^{*}\right|$ was corrected according to the efficiency found for that bin in the PYTHIA and PHOJET samples. The central value is the mean of the result from PYTHIA and PHOJET. The systematic uncertainty on the jet cross-sections given in this section is determined by varying the energy scale of the ECAL in the Monte Carlo simulation by $\pm 5 \%$. The dependence on the Monte Carlo models used is taken into account by adding the difference between the results obtained with PYTHIA and PHOJET to the systematic error. The contributions of these two errors to the total systematic error are of similar size. The systematic errors due to the luminosity measurement, the trigger efficiency and the finite number of Monte Carlo events are small in comparison.

Fig. 3 shows the bin-by-bin corrected $\left|\cos \theta^{*}\right|$ distribution of events with $x_{\gamma}^{ \pm}>0.8$ and of events with $x_{\gamma}^{ \pm}<0.8$. The abscissae of the data points are plotted according to the method proposed in Ref. [26]. The predictions of the theoretical parton distributions are integrated to find the position of the data points. The error of this position is obtained using the predictions of the different parton processes. It is smaller than the line width. The predicted curves have been normalised to the data in the first three bins, in order to compare the shape of the measured cross-sections as a function of $\left|\cos \theta^{*}\right|$ with the QCD matrix element calculation and the NLO QCD calculation The error bars show the statistical and the systematic errors added in quadrature. The overall error on the normalisation of the data points is dominated by the statistical error in the first three bins, which is about $20 \%$. The events with $x_{\gamma}^{ \pm}>0.8$ show a small rise with $\left|\cos \theta^{*}\right|$, whereas the events with $x_{\gamma}^{ \pm}<0.8$ show a much stronger rise with $\left|\cos \theta^{*}\right|$, as expected from the QCD calculations. In the Monte Carlo events about $10 \%$ of the processes with $x_{\gamma}^{ \pm}>0.8$ are double-resolved and about $15 \%$ of the processes with $x_{\gamma}^{ \pm}<0.8$ are direct. The $\left|\cos \theta^{*}\right|$ distribution is not much affected by these impurities, since the Monte Carlo 
double-resolved events with $x_{\gamma}^{ \pm}>0.8$ show a smaller rise with $\left|\cos \theta^{*}\right|$ than the double-resolved events with $x_{\gamma}^{ \pm}<0.8$ and the Monte Carlo direct events with $x_{\gamma}^{ \pm}<0.8$ show a stronger rise with $\left|\cos \theta^{*}\right|$ than the direct events with $x_{\gamma}^{ \pm}>0.8$.

In Fig. 3a the points for $x_{\gamma}^{ \pm}<0.8$ lie close to the predictions of a QCD matrix element calculation of the interaction of quarks or gluons in the photon [2]. The matrix elements with a relevant contribution to the cross-section where anti-quarks are involved instead of quarks show a similar behaviour to the examples shown. The points for $x_{\gamma}^{ \pm}>0.8$ are comparable with the results of a calculation of the process $\gamma \gamma \rightarrow \mathrm{q} \overline{\mathrm{q}}$. The QCD matrix element calculations agree well with the data samples with large direct and large double-resolved contribution.

The data points are compared in Figure $3 \mathrm{~b}$ to a NLO perturbative QCD calculation [3]. The contribution of the different processes to all double-resolved events depends on the parametrisation of the parton distribution functions. This calculation uses the NLO GRV parametrisation and was repeated for the kinematic conditions of this analysis. The shape of the data points and the NLO calculation agrees well. However, before normalisation the predicted cross-section is a factor of two too high for the direct events and about 50\% too small for double-resolved events. A NLO QCD calculation using the GS photon structure function [27] (not shown) shows a similar behaviour.

\section{Jet shapes}

The internal structure of jets produced in photon-photon interactions is studied at the hadron level. The jet shape is characterised by the fraction of a jet's transverse energy $\left(E_{\mathrm{T}}^{\mathrm{jet}}\right)$ that lies inside an inner cone of radius $r$ concentric with the jet defining cone [6]:

$$
\psi(r)=\frac{1}{N_{\text {jet }}} \sum_{\text {jets }} \frac{E_{\mathrm{T}}(r)}{E_{\mathrm{T}}(r=R)},
$$

where $E_{\mathrm{T}}(r)$ is the transverse energy within the inner cone of radius $r$ and $N_{\text {jet }}$ is the total number of jets in the sample. By definition, $\psi(r=R)=1$.

To check the relative importance of parton radiation and fragmentation in the formation of a jet the parton-shower in the LLA as implemented in PYTHIA 5.7 [18] has been used. The jet shape is affected both by fragmentation and gluon radiation.

The jet shapes are corrected to the hadron level using the Monte Carlo event samples with single-resolved, double-resolved and direct processes. The corrected jet shapes are denoted by $\psi(r)$ and refer to jets at the hadron level with a cone radius $R=1$. The reconstructed jet shapes are corrected for acceptance effects and the finite detector resolution. The correction factors also take into account the selection criteria and the purity and efficiency of the jet reconstruction. The corrected jet shapes are determined bin-by-bin as

$$
\psi(r)=C(r) \cdot \psi_{\mathrm{det}}(r)
$$

where the correction factors $C(r)$ are defined as

$$
C(r)=\frac{\psi_{\mathrm{had}}^{\mathrm{MC}}(r)}{\psi_{\mathrm{det}}^{\mathrm{MC}}(r)} .
$$


Since correction factors are different for single-resolved, double-resolved and direct processes, the correction factors have been calculated for each process separately in each $\bar{E}_{\mathrm{T}}^{\text {jet }}$ interval. For direct processes $C(r)$ is close to 1 for all $r$, whereas for single- and double-resolved processes $C(r)$ lies in the range from 0.8 to 1.2 for $r=0.2$ and approaches 1 as $r$ increases. The relative contributions of the different processes have been estimated by comparing the $x_{\gamma}$ distribution of the data events with PYTHIA (see Figure 1) and changing the contributions to get a correct description. In the lowest $\bar{E}_{\mathrm{T}}^{\text {jet }}$ bin $\left(3<\bar{E}_{\mathrm{T}}^{\text {jet }}<6 \mathrm{GeV}\right)$ PYTHIA describes the $x_{\gamma}$ distribution well and the contributions of the different processes were not modified. For the bins in the range $6<\bar{E}_{\mathrm{T}}^{\text {jet }}<20 \mathrm{GeV}$, the contribution of double-resolved events was increased and the contribution of direct events has been reduced, whereas the contribution of single-resolved events was left unchanged. The correction factor $C(r)$ in each $\bar{E}_{\mathrm{T}}^{\text {jet }}$ bin is calculated according to the relative contribution of each process determined in this way.

The $\bar{E}_{\mathrm{T}}^{\mathrm{jet}}$ dependence of the jet shapes in di-jet production is presented in Figure $\sharp$. The predictions of PYTHIA for direct, single-resolved and double-resolved processes and their sum are compared to the measured jet shapes. The central value of the plotted data points is obtained with the correction calculated from PYTHIA. The systematic error was obtained from the variation of the ECAL energy by $\pm 5 \%$ and from the difference of the results from PYTHIA and PHOJET. The relative contribution of the processes in PYTHIA has also been changed over a range consistent with what the match to Figure 11 will allow, and the resulting changes in the corrections have been added to the systematic error. The jets become narrower as $\bar{E}_{\mathrm{T}}^{\text {jet }}$ increases. The predictions of PYTHIA with the default relative contributions of the different processes using the SaS-1D parametrisation reproduce the data reasonably well. There is almost no difference between the predictions of PYTHIA and PHOJET using GRV (not shown). The differences of the jet shapes for direct, single- and double-resolved processes are expected to be due to the different fractions of quark and gluon jets. It has been shown that gluon jets are broader than quark jets [17]. According to the prediction of PYTHIA using SaS-1D the fraction of gluon jets is $12 \%$ for direct events, $16 \%$ for single-resolved events and $41 \%$ for double-resolved events.

The fraction of the transverse energy of the jets inside a cone of radius $r=0.5$ around the jet axis, $\psi(r=0.5)$ is shown as a function of $\bar{E}_{\mathrm{T}}^{\text {jet }}$ (Figure 5a) and as a function of $\left|\eta^{\text {jet }}\right|$ (Figure 5b). The position of the data points is the mean value of $\bar{E}_{\mathrm{T}}^{\text {jet }}$ in each bin. In Figure 5a the data are compared to PYTHIA with and without multiple interactions and to PYTHIA without initial (ISR) and final state QCD radiation (FSR). The PHOJET prediction is also shown. The difference between PYTHIA with and without multiple interactions (mi) is very small, whereas the PYTHIA prediction without initial and final state QCD radiation, where only fragmentation effects have been taken into account, leads to jets which are significantly narrower. This difference increases with increasing $\bar{E}_{\mathrm{T}}^{\mathrm{jet}}$. Fig. 5b shows that there is no observed dependence of the jet shape on $\left|\eta^{\text {jet }}\right|$. The prediction of PYTHIA is in good agreement with the data. Gluon jets are predicted to be broader than quark jets. The hadron jets in the Monte Carlo events have been identified as a quark or gluon jet depending on the type (quark or gluon) of the closest parton in the $\eta \phi$ plane.

The results for $\psi(r)$ are presented in Figure 5c and d for both $x_{\gamma}^{ \pm}$smaller and larger than 0.8. It is observed that the measured jet shapes for $x_{\gamma}^{ \pm}<0.8$, where more gluon jets are expected, are broader than those for $x_{\gamma}^{ \pm}>0.8$. For both regions of $x_{\gamma}^{ \pm}$the Monte Carlo generators reproduce the data reasonably well. 


\section{Inclusive two-jet cross-sections and NLO calculations}

To obtain absolute jet cross-sections which can be compared to theoretical calculations, we use the Monte Carlo simulation and an unfolding program [28] to correct for the selection cuts, the resolution effects of the detector and the background from non-signal processes. The same technique was used as described in Ref. [1]. To improve the performance of the unfolding program in the region $E_{\mathrm{T}}^{\text {jet }}>3 \mathrm{GeV}$, bin-to-bin migration effects from jets at low $E_{\mathrm{T}}^{\text {jet }}$ must be taken into account. Therefore the jets are actually found with $E_{\mathrm{T}}^{\mathrm{min}}=2 \mathrm{GeV}$ and the unfolding is performed in the full $E_{\mathrm{T}}^{\mathrm{jet}}>2 \mathrm{GeV}$ range, but the unfolded jet cross-section are only shown for $E_{\mathrm{T}}^{\text {jet }}>3 \mathrm{GeV}$. The uncorrected number of jets reconstructed in the range $3<E_{\mathrm{T}}^{\text {jet }}<4 \mathrm{GeV}$ is about $15 \%$ larger with $E_{\mathrm{T}}^{\min }=3 \mathrm{GeV}$ than with $E_{\mathrm{T}}^{\min }=2 \mathrm{GeV}$. This difference decreases to less than $5 \%$ for higher $E_{\mathrm{T}}^{\text {jet }}$.

In Fig. 6, the inclusive two-jet cross-section is shown as a function of $E_{\mathrm{T}}^{\text {jet }}$. The average transverse energy, $\left\langle E_{\mathrm{T}}^{\mathrm{jet}}\right\rangle$, within each bin, which is plotted on the abscissa, is determined as proposed in Ref. [26]. It is obtained by integrating an exponential function which is fitted to the neighbouring data points. The error on $\left\langle E_{\mathrm{T}}^{\mathrm{jet}}\right\rangle$ is calculated by varying the slope of the exponential function. The error bars show the statistical and the systematic errors, calculated in the same way as in Section 7, and an additional error from the unfolding procedure are added in quadrature. The bin sizes, which are indicated by the tic marks at the top of the Figures, approximately reflect the experimental resolution. The results are summarised in Table 2 .

The $E_{\mathrm{T}}^{\mathrm{jet}}$ distribution is compared to a NLO perturbative QCD calculation of the inclusive two-jet cross-section by Kleinwort and Kramer [10] who use the NLO GRV parametrisation of the parton distribution functions of the photon [22]. Their calculation was repeated for the kinematic conditions of this analysis. The renormalisation and factorisation scales are chosen to be equal to $E_{\mathrm{T}}^{\mathrm{jet}}$. The scale dependence of the NLO QCD calculations is expected to be small [3]. The strong coupling constant $\alpha_{\mathrm{s}}$ is calculated from the two-loop formula with $\Lambda \frac{(5)}{\mathrm{MS}}=130 \mathrm{MeV}$, since this value is also used in the NLO-GRV parametrisation. Changing $\Lambda \frac{(5)}{\mathrm{MS}}$ from 130 to 250 $\mathrm{MeV}$ only in the $\alpha_{\mathrm{s}}$ formula increases the two-jet cross-section by factors from 1.4 to 1.07 in the range $3<E_{\mathrm{T}}^{\text {jet }}<16 \mathrm{GeV}$ for $\sqrt{s}_{\text {ee }}=130-136 \mathrm{GeV}$ [29].

The data points are in good agreement with the calculation except in the first bin where the calculation predicts a much higher cross-section. The symmetric cuts on $E_{\mathrm{T}}^{\mathrm{jet}}$ lead to singularities of the NLO calculations. This problem only affects the first bin, where the NLO calculations are not reliable. The NLO QCD calculation gives the jet cross-section for massless partons, whereas the experimental jet cross-sections are measured for hadrons. The uncertainties due to the modelling of the hadronisation process have not been taken into account. Because the partons in the Monte Carlo models and the partons in the NLO calculations are defined in different ways it is impossible to use the Monte Carlo to correct the data so that it can be compared with the NLO parton level predictions. If PYTHIA had been used to calculate a correction we would have had to increase the cross-section by a factor of between 1.2 and 1.3, with the largest effects at low $E_{\mathrm{T}}^{\text {jet }}$.

The predictions for the direct, single- and double-resolved parts and their sum are shown separately. The resolved cross-sections is the largest component in the region $E_{\mathrm{T}}^{\text {jet }} \lesssim 8 \mathrm{GeV}$, whereas, at high $E_{\mathrm{T}}^{\mathrm{jet}}$ the direct cross-section is largest. 


\section{Influence of the underlying event}

The NLO QCD calculations also do not take into account the possibility of an underlying event which leads to an increased jet cross-section. The underlying event is simulated in the Monte Carlo models PYTHIA and PHOJET which will be used to compare to different LO parametrisations of the parton distribution, SaS-1D [21], GRV [22] and LAC1 [32]. These sets of parton distributions contain different parametrisations of the gluon density with LAC1 predicting a much larger gluon density than GRV and SaS-1D. In PYTHIA and PHOJET the modelling of the underlying event includes multiple interactions. The contribution from multiple interactions has to be tuned using quantities which are not directly correlated to the jets, since otherwise effects of the parton distributions and of the underlying event cannot be distinguished. A significant difference between the predicted two-jet cross-sections obtained with PYTHIA and PHOJET using the same parametrisation of the parton distributions was observed in our studies at lower energies [1]. By adjusting the cutoff parameter $p_{\mathrm{t}}^{\mathrm{mi}}$ for multiple interactions the model dependence should decrease significantly.

It is expected that the transverse energy flow outside the jets measured as a function of $x_{\gamma}$ is correlated to the underlying event [30]. No effect due to the underlying event is expected for direct events at large $x_{\gamma}$. The increase of the transverse energy flow outside the two jets at small $x_{\gamma}$ can therefore be used to tune the number of multiple interactions in the model.

The events were boosted into their centre-of-mass system and the transverse energy flow was measured as a function of $x_{\gamma}$ in the central rapidity region $\left|\eta^{*}\right|<1$. The regions around the jet axes with $R<1.3$ are excluded from the energy sum. As in Section 6, $x_{\gamma}$ denotes that the transverse energy flow of each event is added to the plot at the values of $x_{\gamma}^{+}$and of $x_{\gamma}^{-}$. Fig. 7 shows the transverse energy flows corrected to the hadron level. The systematic error was obtained from the difference of the results from PYTHIA and PHOJET and from the variation of the ECAL energy by $\pm 5 \%$. Fig. 7a shows the results of PYTHIA using the LAC1 parametrisation with different $p_{\mathrm{t}}^{\mathrm{mi}}$ cutoff parameters. The transverse energy flow for the default $p_{\mathrm{t}}^{\mathrm{mi}}$ of $1.4 \mathrm{GeV} / c$ is much too high in the first $x_{\gamma}$ bins. Without multiple interactions the transverse energy flow is too low. An optimised value of $p_{\mathrm{t}}^{\mathrm{mi}}=2.5 \mathrm{GeV} / c$ leads to a reasonable description of the data. The average number $n_{\mathrm{mi}}$ of interactions in double-resolved di-jet events is 4.1 for $\mathrm{LAC} 1$ with $p_{\mathrm{t}}^{\mathrm{mi}}=1.4 \mathrm{GeV} / c$ and 1.5 with $p_{\mathrm{t}}^{\mathrm{mi}}=2.5 \mathrm{GeV} / c$.

The difference of the transverse energy flow using SaS-1D in PYTHIA with and without multiple interactions $\left(p_{\mathrm{t}}^{\mathrm{mi}}=1.4 \mathrm{GeV} / c\right)$ is very small and the predicted transverse energy flow is in good agreement with the data. The best description using PYTHIA and GRV is obtained with a $p_{\mathrm{t}}^{\mathrm{mi}}$ of $2.0 \mathrm{GeV} / c$. For all further comparisons with PYTHIA, the cutoff parameter $p_{\mathrm{t}}^{\mathrm{mi}}$ was set to $2.5 \mathrm{GeV} / c$ for LAC1, to $2.0 \mathrm{GeV} / c$ for GRV and to $1.4 \mathrm{GeV} / c$ for SaS-1D.

In $\gamma \mathrm{p}$ collisions at HERA the GRV parametrisation with a cutoff parameter of $p_{\mathrm{t}}^{\mathrm{mi}}=$ $1.2 \mathrm{GeV} / c$ for PYTHIA has been found to be in good agreement with the data, whereas the cutoff parameter was set to $2.0 \mathrm{GeV} / c$ for LAC1 [30]. With this choice the models slightly overestimate the transverse energy flows at low $x_{\gamma}$ in our data.

PHOJET with either SaS-1D or GRV is in reasonable agreement with the data. Changing the default cutoff of PHOJET from $p_{\mathrm{t}}^{\mathrm{mi}}=2.5 \mathrm{GeV} / c$ does not affect the transverse energy flow significantly, the parameter $p_{\mathrm{t}}^{\mathrm{mi}}$ was therefore left unchanged in PHOJET. 


\section{Inclusive two-jet cross-sections as function of $\left|\eta^{\text {jet }}\right|$}

The size and $\left|\eta^{\text {jet}}\right|$ dependence of the inclusive two-jet cross-section, which is dominated by the low $E_{\mathrm{T}}^{\mathrm{jet}}$ events, depend on the chosen parton distribution functions which mainly differ in the assumptions on the gluonic content of the photon. This leads to different predictions for the inclusive two-jet cross-section, especially for double-resolved events. The inclusive two-jet cross-section as a function of $\left|\eta^{\text {jet }}\right|$ is shown in Figure 8 for events with $E_{\mathrm{T}}^{\text {jet1 }}>4 \mathrm{GeV}$ and $E_{\mathrm{T}}^{\text {jet2 }}>3 \mathrm{GeV}$ and in Figure 9 for events with $E_{\mathrm{T}}^{\text {jet1 }}>5 \mathrm{GeV}$ and $E_{\mathrm{T}}^{\mathrm{jet} 2}>3 \mathrm{GeV}$. The asymmetric cuts have been applied because NLO calculations with symmetric cuts are not infrared safe [31].

The data sample is separated into events with a large contribution from double-resolved processes by requiring $x_{\gamma}^{ \pm}<0.8$ (Fig. $8 \mathrm{~b}$ and $9 \mathrm{~b}$ ) and into events with a large contribution from direct processes by requiring $x_{\gamma}^{ \pm}>0.8$ (Fig. $8 \mathrm{c}$ and $9 \mathrm{c}$ ). The results are summarised in Tables 3 and 1 . The average $\left\langle\left|\eta^{\text {jet }}\right|\right\rangle$ values are consistent with being at the centre of the bins. Each jet is included with its value of $\left|\eta^{\text {jet }}\right|$ in the cross-section measurement. Within the statistical and systematic uncertainties of the measurement, the data distributions are nearly independent of $\left|\eta^{\text {jet }}\right|$ in Fig. 8 $\mathrm{a}$ and b with a small drop towards $\left|\eta^{\text {jet }}\right|=2$, whereas the data distribution of the direct events shown in Fig. $8 \mathrm{c}$ at $\left|\eta^{\text {jet }}\right|=2$ falls to about half of its value at $\left|\eta^{\text {jet }}\right|=0$. In the kinematic range shown, this is in agreement with the expectations of the Monte Carlo models.

The NLO QCD calculation of the inclusive two-jet cross-section is in excellent agreement with the differential cross-section $\mathrm{d} \sigma / \mathrm{d}\left|\eta^{\text {jet }}\right|$ shown in Figs. 8a and 9a. The SaS-1D parametrisation [21] with the PYTHIA and PHOJET models predicts a two-jet cross-section which is significantly too low for the whole data sample and for events with a large contribution from double-resolved events $\left(x_{\gamma}^{ \pm}<0.8\right)$. The cross-section using GRV [22] is a bit too low for the whole data sample but is able to describe the cross-section for the events with $x_{\gamma}^{ \pm}<0.8$. In this range the cross-sections as predicted using LAC1 [32]. are much too high. It should be noted that the overall normalisation of jet cross-sections can alway be adjusted in a LO calculation by changing the value of the strong coupling $\alpha_{\mathrm{s}}$.

In contrast to these discrepancies between the predicted cross-sections for $x_{\gamma}^{ \pm}<0.8$ due to different parametrisations of the parton distributions, the differences between the predicted cross-sections for events with a large contribution of direct events $\left(x_{\gamma}^{ \pm}>0.8\right)$ seem to depend mainly on the model, PHOJET or PYTHIA, and not the parametrisation used (Figs. 8c and 9c). The PHOJET cross-section is slightly too high, whereas PYTHIA describes the data well. As expected, this is independent of the chosen cutoff parameter for multiple interactions.

To further reduce the influence from multiple interactions and hadronisation effects the inclusive two-jet cross-section was also measured for events with $E_{\mathrm{T}}^{\mathrm{jet}}>5 \mathrm{GeV}$. (Fig. 10). The results are summarised in Table 5. The fraction of resolved events is smaller in this data sample and the difference between the different parametrisations decreases. Nevertheless the LAC1 prediction is still too high for the whole data sample and especially for the data sample with a large contribution of double-resolved events (Fig. 10 b). The fraction of the cross-section with $x_{\gamma}^{ \pm}>0.8$ compared to the total jet cross-section is significantly larger in the range $E_{\mathrm{T}}^{\text {jet }}>5 \mathrm{GeV}$ than for the lower $E_{\mathrm{T}}^{\mathrm{jet}}$ ranges. 


\section{Conclusions}

We have measured di-jet production in photon-photon interactions with the OPAL detector at $\mathrm{e}^{+} \mathrm{e}^{-}$centre-of-mass energies $\sqrt{s}_{\text {ee }}$ of 161 and $172 \mathrm{GeV}$ with an integrated luminosity of $20 \mathrm{pb}^{-1}$. Jets were identified using a cone jet finding algorithm with $R=1$ in the kinematic range $E_{\mathrm{T}}^{\text {jet }}>3 \mathrm{GeV}$ and $\left|\eta^{\text {jet }}\right|<2$. Two-jet events originating mainly from direct and doubleresolved photon interactions were separated experimentally using the variables $x_{\gamma}^{+}$and $x_{\gamma}^{-}$. The Monte Carlo models PYTHIA and PHOJET describe the transverse energy flow around the jets reasonably well.

The distribution of the parton scattering angle $\theta^{*}$ has been reconstructed from the rapidities of the two jets. Data samples with large direct and double-resolved contributions have been compared to LO and NLO QCD calculations. A strong rise has been observed in the $\left|\cos \theta^{*}\right|$ distribution of the data sample with a large double-resolved contribution at high $\left|\cos \theta^{*}\right|$, as expected from QCD. The flatter $\left|\cos \theta^{*}\right|$ distribution of the data sample with a large contribution from direct events is also in good agreement with the QCD calculation.

The energy profile of the jets has been measured in different regions of $\bar{E}_{\mathrm{T}}^{\mathrm{jet}}$. It has been observed that jets with high $\bar{E}_{\mathrm{T}}^{\text {jet }}$ are narrower than jets with small values of $\bar{E}_{\mathrm{T}}^{\text {jet }}$. The jet shape in events with $x_{\gamma}^{ \pm}<0.8$, where double-resolved events are expected to dominate, is found to be broader than the jet shape in events with $x_{\gamma}^{ \pm}>0.8$, where direct events are expected to dominate. These differences are assumed to be caused by different fractions of quark jets and gluon jets. The influence of multiple interactions and of different parametrisations of the parton distribution functions of the photon is small.

The inclusive two-jet cross-sections were measured as a function of $E_{\mathrm{T}}^{\text {jet }}$ and $\left|\eta^{\text {jet }}\right|$. The measured cross-sections are in good agreement with next-to-leading order QCD calculations by Klasen, Kleinwort and Kramer [10] above $E_{\mathrm{T}}^{\text {jet }}=4 \mathrm{GeV}$ using the NLO GRV parametrisation of the parton distributions of the photon.

The inclusive two-jet cross-section is dominated by the resolved processes in the low $E_{\mathrm{T}}^{\text {jet }}$ region. In order to distinguish between the contributions to the jet cross-section from possible multiple interactions between the spectator partons and from the parton densities, the contribution of multiple interactions in the models has been tuned using the measured transverse energy flow outside the jets at low $x_{\gamma}$. However, within the errors of the measurement we are unable to differentiate between models with and without multiple interactions.

Using PYTHIA and PHOJET the LO GRV parametrisation is also able to describe the two-jet cross-section whereas the cross-section predicted based on the SaS-1D parametrisation is too low and the prediction based on the LAC1 parametrisation is significantly too high. The same behaviour is observed using a data sample with a large contribution from doubleresolved events. As expected, there is no sensitivity to the choice of parametrisation for the complementary data sample with a large contribution from direct events. This behaviour still holds if the inclusive two-jet cross-sections are measured for $E_{\mathrm{T}}^{\mathrm{jet}}>5 \mathrm{GeV}$. 


\section{Acknowledgements}

We thank R. Engel and T. Sjöstrand for providing the Monte Carlo code and for many useful discussions and M. Klasen, T. Kleinwort and G. Kramer for providing the NLO calculations. We particularly wish to thank the SL Division for the efficient operation of the LEP accelerator at all energies and for their continuing close cooperation with our experimental group. We thank our colleagues from CEA, DAPNIA/SPP, CE-Saclay for their efforts over the years on the time-of-flight and trigger systems which we continue to use. In addition to the support staff at our own institutions we are pleased to acknowledge the

Department of Energy, USA, National Science Foundation, USA, Particle Physics and Astronomy Research Council, UK, Natural Sciences and Engineering Research Council, Canada, Israel Science Foundation, administered by the Israel Academy of Science and Humanities, Minerva Gesellschaft, Benoziyo Center for High Energy Physics, Japanese Ministry of Education, Science and Culture (the Monbusho) and a grant under the Monbusho International Science Research Program, German Israeli Bi-national Science Foundation (GIF), Bundesministerium für Bildung, Wissenschaft, Forschung und Technologie, Germany, National Research Council of Canada, Research Corporation, USA, Hungarian Foundation for Scientific Research, OTKA T-016660, T023793 and OTKA F-023259. 


\section{References}

[1] OPAL Collaboration, K. Ackerstaff et al., Z. Phys. C73 (1997) 433.

[2] for example: H. Kolanoski, Two-Photon Physics at $e^{+} e^{-}$Storage Rings, Springer-Verlag (1984);

B.L. Combridge et al., Phys. Lett. B70 (1977) 234;

D.W. Duke and J. F. Owens, Phys. Rev. D26 (1982) 1600.

[3] M. Klasen, T. Kleinwort and G. Kramer, Inclusive Jet Production in $\gamma p$ and $\gamma \gamma$ Processes: Direct and Resolved Photon Cross Sections in Next-To-Leading Order QCD, DESY-97-234 and hep-ph/9712256 (1997);

M. Klasen, T. Kleinwort and G. Kramer, private communication.

[4] CDF Collaboration, F. Abe et al., Phys. Rev. Lett. 77 (1996) 5336.

[5] ZEUS Collaboration, M. Derrick et al., Phys. Lett. B384 (1996) 388;

ZEUS Collaboration, M. Derrick et al., Phys. Lett. B348 (1995) 665.

[6] S.D. Ellis, Z. Kunszt and D.E. Soper, Phys. Rev. Lett. 69 (1992) 3615.

[7] ZEUS Collaboration, J. Breitweg et al., Eur. Phys. J. C2 (1998) 61.

[8] AMY Collaboration, B.J. Kim et al., Phys. Lett. B325 (1994) 248.

[9] ZEUS Collaboration, J. Breitweg et al., High-E $E_{\mathrm{T}}$ Inclusive Jet Cross Sections in Photoproduction at HERA, DESY-98-018 and hep-ex/9802012 (1998);

ZEUS Collaboration, J. Breitweg et al., Eur. Phys. J. C1 (1998) 109;

H1 Collaboration, C. Adloff et al., Eur. Phys. J. C1 (1998) 97.

[10] T. Kleinwort, G. Kramer, Nucl. Phys. B477 (1996) 3;

T. Kleinwort, G. Kramer, Phys. Lett. B370 (1996) 141;

M. Klasen, T. Kleinwort, G. Kramer, private communication.

[11] P. Aurenche et al., Progr. Theor. Phys. 92 (1994) 175;

P. Aurenche et al., Proc. Workshop on Two-Photon Physics at LEP and HERA, Lund, Sweden (May 1994) 269.

[12] OPAL Collaboration, R. Akers et al., Z. Phys. C61 (1994) 199;

OPAL Collaboration, K. Ackerstaff et al., Z. Phys. C74 (1997) 33;

DELPHI Collaboration, P. Abreu et al., Z. Phys. C69 (1996) 223.

[13] TOPAZ Collaboration, H. Hayashii et al., Proc. 10th Workshop on Gamma-Gamma Collisions and Related Processes, Sheffield, UK (April 1995) 133;

TOPAZ Collaboration, H. Hayashii et al., Phys. Lett. B314 (1993) 149.

[14] For a recent discussion see: G.A. Schuler, Improving the equivalent-photon approximation in electron-positron collisions, CERN-TH/96-297 and hep-ph/9610406.

[15] OPAL Collaboration, K. Ahmet et al., Nucl. Instrum. Methods A305 (1991) 275;

O. Biebel et al., Nucl. Instrum. Methods A323 (1992) 169;

S. Anderson et al., Nucl. Instrum. Methods A403 (1998) 326. 
[16] B.E. Anderson et al., IEEE Transactions on Nuclear Science 41 (1994) 845.

[17] OPAL Collaboration, R. Akers et al., Z. Phys. C63 (1994) 197.

[18] T. Sjöstrand, Comp. Phys. Comm. 82 (1994) 74;

T. Sjöstrand, LUND University Report, LU-TP-95-20 (1995).

[19] G.A. Schuler and T. Sjöstrand, Z. Phys. C73 (1997) 677;

G.A. Schuler and T. Sjöstrand, Nucl. Phys. B407 (1993) 539.

[20] R. Engel and J. Ranft, Phys. Rev. D54 (1996) 4244;

R. Engel, Z. Phys. C66 (1995) 203.

[21] G.A. Schuler and T. Sjöstrand, Z. Phys. C68 (1995) 607.

[22] M. Glück, E. Reya and A. Vogt, Phys. Rev. D46 (1992) 1973;

M. Glück, E. Reya and A. Vogt, Phys. Rev. D45 (1992) 3986.

[23] J. Allison et al., Nucl. Instrum. Methods A317 (1992) 47.

[24] OPAL Collaboration, K. Ackerstaff et al., Inclusive Production of Charged Hadrons and $K_{\mathrm{s}}^{0}$ Mesons in Photon-Photon Collisions, CERN-EP/98-091 (1998).

[25] L. Lönnblad and M. Seymour (convenors), $\gamma \gamma$ Event Generators, in "Physics at LEP2", CERN 96-01, eds. G. Altarelli, T. Sjöstrand and F. Zwirner, Vol. 2 (1996) 187.

[26] G.D. Lafferty and T.R. Wyatt, Nucl. Instrum. Methods A355 (1995) 541.

[27] L.E. Gordon and J.K. Storrow, Phys. Lett. B291 (1994) 320.

[28] V. Blobel, DESY-84-118 (1984);

V. Blobel, Proc. 1984 CERN School of Computing, Aiguablava, Spain, CERN 85-09 (September 1984);

V. Blobel, The RUN manual, Regularized Unfolding for High-Energy Physics Experiments, program manual, unpublished.

[29] T. Kleinwort, G. Kramer, Z. Phys. C75 (1997) 489.

[30] H1 Collaboration, S. Aid et al., Z. Phys. C70 (1996) 17.

[31] M. Klasen and G. Kramer, Phys. Lett. B366 (1996) 385.

[32] H. Abramowicz, K. Charchula and A. Levy, Phys. Lett. B269 (1991) 458. 


\begin{tabular}{|c|c|r|}
\hline \multirow{2}{*}{$\left|\cos \theta^{*}\right|$} & \multicolumn{2}{|c|}{$\mathrm{d} \sigma / \mathrm{d}\left|\cos \theta^{*}\right|[\mathrm{pb}]$} \\
\cline { 2 - 3 } & resolved $\left(x_{\gamma}^{ \pm}<0.8\right)$ & \multicolumn{1}{|c|}{$\operatorname{direct}\left(x_{\gamma}^{ \pm}>0.8\right)$} \\
\hline $0.000-0.106$ & $6.2 \pm 1.7 \pm 0.3$ & $4.2 \pm 1.4 \pm 0.2$ \\
$0.106-0.213$ & $2.7 \pm 1.1 \pm 0.2$ & $5.0 \pm 1.5 \pm 0.4$ \\
$0.213-0.319$ & $9.7 \pm 2.1 \pm 1.1$ & $5.6 \pm 1.6 \pm 0.4$ \\
$0.319-0.425$ & $10.3 \pm 2.2 \pm 2.3$ & $7.8 \pm 1.9 \pm 0.1$ \\
$0.425-0.531$ & $10.5 \pm 2.2 \pm 1.7$ & $6.8 \pm 1.8 \pm 0.5$ \\
$0.531-0.638$ & $22.7 \pm 3.3 \pm 2.4$ & $7.2 \pm 1.8 \pm 0.4$ \\
$0.638-0.744$ & $30.1 \pm 3.8 \pm 2.5$ & $6.5 \pm 1.7 \pm 0.1$ \\
$0.744-0.850$ & $51.9 \pm 5.0 \pm 3.6$ & $15.8 \pm 2.7 \pm 0.7$ \\
\hline
\end{tabular}

Table 1: Differential two-jet cross-section as a function of $\left|\cos \theta^{*}\right|$. The cross section is shown for events with $x_{\gamma}^{ \pm}<0.8$ and for events with $x_{\gamma}^{ \pm}>0.8$. The first error is statistical and the second error is systematic.

\begin{tabular}{|r|r|ccl|}
\hline$E_{\mathrm{T}}^{\text {jet }}(\mathrm{GeV})$ & $\left\langle E_{\mathrm{T}}^{\text {jet }}\right\rangle(\mathrm{GeV})$ & \multicolumn{2}{|c|}{$\mathrm{d} \sigma / \mathrm{d} E_{\mathrm{T}}^{\text {jet }}[\mathrm{pb} / \mathrm{GeV}]$} \\
\hline $3.0-4.0$ & $3.47 \pm 0.01$ & 163 & \pm 3 & \pm 16 \\
$4.0-5.0$ & $4.47 \pm 0.01$ & 73.6 & \pm 2.0 & \pm 8.7 \\
$5.0-6.5$ & $5.69 \pm 0.01$ & 27.9 & \pm 0.9 & \pm 3.9 \\
$6.5-8.5$ & $7.42 \pm 0.01$ & 11.5 & \pm 0.5 & \pm 1.4 \\
$8.5-11.0$ & $9.64 \pm 0.01$ & 3.83 & $\pm 0.26 \pm 0.60$ \\
$11.0-15.0$ & $12.73 \pm 0.02$ & $1.30 \pm 0.14 \pm 0.35$ \\
$15.0-20.0$ & $17.16 \pm 0.02$ & $0.12 \pm 0.04 \pm 0.13$ \\
\hline
\end{tabular}

Table 2: The inclusive two-jet cross-section as a function of $E_{\mathrm{T}}^{\mathrm{jet}}$. The first error is statistical and the second error is systematic. 


\begin{tabular}{|c|r|c|c|}
\hline \multirow{2}{*}{$\left|\eta^{\text {jet }}\right|$} & \multicolumn{3}{|c|}{$\mathrm{d} \sigma / \mathrm{d}\left|\eta^{\text {jet }}\right|[\mathrm{pb}]$} \\
\cline { 2 - 4 } & \multicolumn{1}{|c|}{ no $x_{\gamma}^{ \pm}$cut } & resolved $\left(x_{\gamma}^{ \pm}<0.8\right)$ & direct $\left(x_{\gamma}^{ \pm}>0.8\right)$ \\
\hline $0.0-0.4$ & $118.7 \pm 3.7 \pm 12.8$ & $77.2 \pm 3.3 \pm 11.8$ & $15.3 \pm 1.2 \pm 2.5$ \\
$0.4-0.8$ & $114.4 \pm 3.6 \pm 10.7$ & $73.3 \pm 3.1 \pm 10.4$ & $15.1 \pm 1.2 \pm 2.4$ \\
$0.8-1.2$ & $93.4 \pm 2.9 \pm 11.7$ & $57.2 \pm 2.5 \pm 10.9$ & $13.6 \pm 1.1 \pm 2.4$ \\
$1.2-1.6$ & $95.1 \pm 3.5 \pm 11.7$ & $56.0 \pm 2.8 \pm 11.5$ & $10.9 \pm 1.3 \pm 2.2$ \\
$1.6-2.0$ & $81.2 \pm 3.8 \pm 9.7$ & $60.5 \pm 3.4 \pm 10.9$ & $8.2 \pm 1.2 \pm 1.9$ \\
\hline
\end{tabular}

Table 3: The inclusive two-jet cross-section as a function of $\left|\eta^{\mathrm{jet}}\right|$ for events with $E_{\mathrm{T}}^{\mathrm{jet} 1}>4 \mathrm{GeV}$ and $E_{\mathrm{T}}^{\mathrm{jet} 2}>3 \mathrm{GeV}$. The inclusive two-jet cross-section is shown for all two-jet events and for data samples with a large contribution from double-resolved events by requiring $x_{\gamma}^{ \pm}<0.8$ and with a large contribution from direct events by requiring $x_{\gamma}^{ \pm}>0.8$. The first error is statistical and the second error is systematic.

\begin{tabular}{|c|c|c|c|}
\hline \multirow{2}{*}{$\left|\eta^{\text {jet }}\right|$} & \multicolumn{3}{|c|}{$\mathrm{d} \sigma / \mathrm{d}\left|\eta^{\text {jet }}\right|[\mathrm{pb}]$} \\
\cline { 2 - 4 } & no $x_{\gamma}^{ \pm}$cut & resolved $\left(x_{\gamma}^{ \pm}<0.8\right)$ & direct $\left(x_{\gamma}^{ \pm}>0.8\right)$ \\
\hline $0.0-0.4$ & $58.3 \pm 2.6 \pm 7.7$ & $35.0 \pm 2.3 \pm 6.5$ & $8.8 \pm 0.8 \pm 1.9$ \\
$0.4-0.8$ & $57.8 \pm 2.4 \pm 7.6$ & $33.6 \pm 2.0 \pm 6.0$ & $9.5 \pm 0.9 \pm 1.8$ \\
$0.8-1.2$ & $49.8 \pm 2.0 \pm 6.5$ & $31.3 \pm 2.0 \pm 6.7$ & $9.2 \pm 0.9 \pm 1.9$ \\
$1.2-1.6$ & $52.8 \pm 2.4 \pm 6.6$ & $28.8 \pm 2.0 \pm 5.8$ & $7.9 \pm 1.0 \pm 1.7$ \\
$1.6-2.0$ & $45.2 \pm 2.9 \pm 7.2$ & $30.2 \pm 2.3 \pm 6.2$ & $5.9 \pm 1.0 \pm 1.5$ \\
\hline
\end{tabular}

Table 4: The inclusive two-jet cross-section as a function of $\left|\eta^{\mathrm{jet}}\right|$ for events with $E_{\mathrm{T}}^{\mathrm{jet} 1}>5 G e V$ and $E_{\mathrm{T}}^{\mathrm{jet} 2}>3 \mathrm{GeV}$. The inclusive two-jet cross-section is shown for all two-jet events and for data samples with a large contribution from double-resolved events by requiring $x_{\gamma}^{ \pm}<0.8$ and with a large contribution from direct events by requiring $x_{\gamma}^{ \pm}>0.8$. The first error is statistical and the second error is systematic.

\begin{tabular}{|c|c|c|c|}
\hline \multirow{2}{*}{$\left|\eta^{\text {jet }}\right|$} & \multicolumn{3}{|c|}{$\mathrm{d} \sigma / \mathrm{d}\left|\eta^{\text {jet }}\right|[\mathrm{pb}]$} \\
\cline { 2 - 4 } & no $x_{\gamma}^{ \pm}$cut & resolved $\left(x_{\gamma}^{ \pm}<0.8\right)$ & $\operatorname{direct}\left(x_{\gamma}^{ \pm}>0.8\right)$ \\
\hline $0.0-0.4$ & $31.0 \pm 1.4 \pm 3.5$ & $17.2 \pm 1.1 \pm 4.4$ & $8.3 \pm 0.9 \pm 1.7$ \\
$0.4-0.8$ & $32.0 \pm 1.6 \pm 3.4$ & $15.6 \pm 1.1 \pm 2.9$ & $9.3 \pm 1.0 \pm 1.8$ \\
$0.8-1.2$ & $27.6 \pm 1.4 \pm 3.2$ & $11.3 \pm 0.9 \pm 3.3$ & $9.2 \pm 1.0 \pm 1.7$ \\
$1.2-1.6$ & $27.5 \pm 1.6 \pm 5.8$ & $11.0 \pm 0.9 \pm 3.1$ & $7.8 \pm 1.0 \pm 1.6$ \\
$1.6-2.0$ & $18.3 \pm 1.5 \pm 3.1$ & $7.5 \pm 0.9 \pm 3.3$ & $5.8 \pm 1.2 \pm 1.5$ \\
\hline
\end{tabular}

Table 5: The inclusive two-jet cross-section as a function of $\left|\eta^{\mathrm{jet}}\right|$ for events with $E_{\mathrm{T}}^{\mathrm{jet}}>5 \mathrm{GeV}$. The inclusive two-jet cross-section is shown for all two-jet events and for data samples with a large contribution from double-resolved events by requiring $x_{\gamma}^{ \pm}<0.8$ and with a large contribution from direct events by requiring $x_{\gamma}^{ \pm}>0.8$. The first error is statistical and the second error is systematic. 


\section{OPAL}
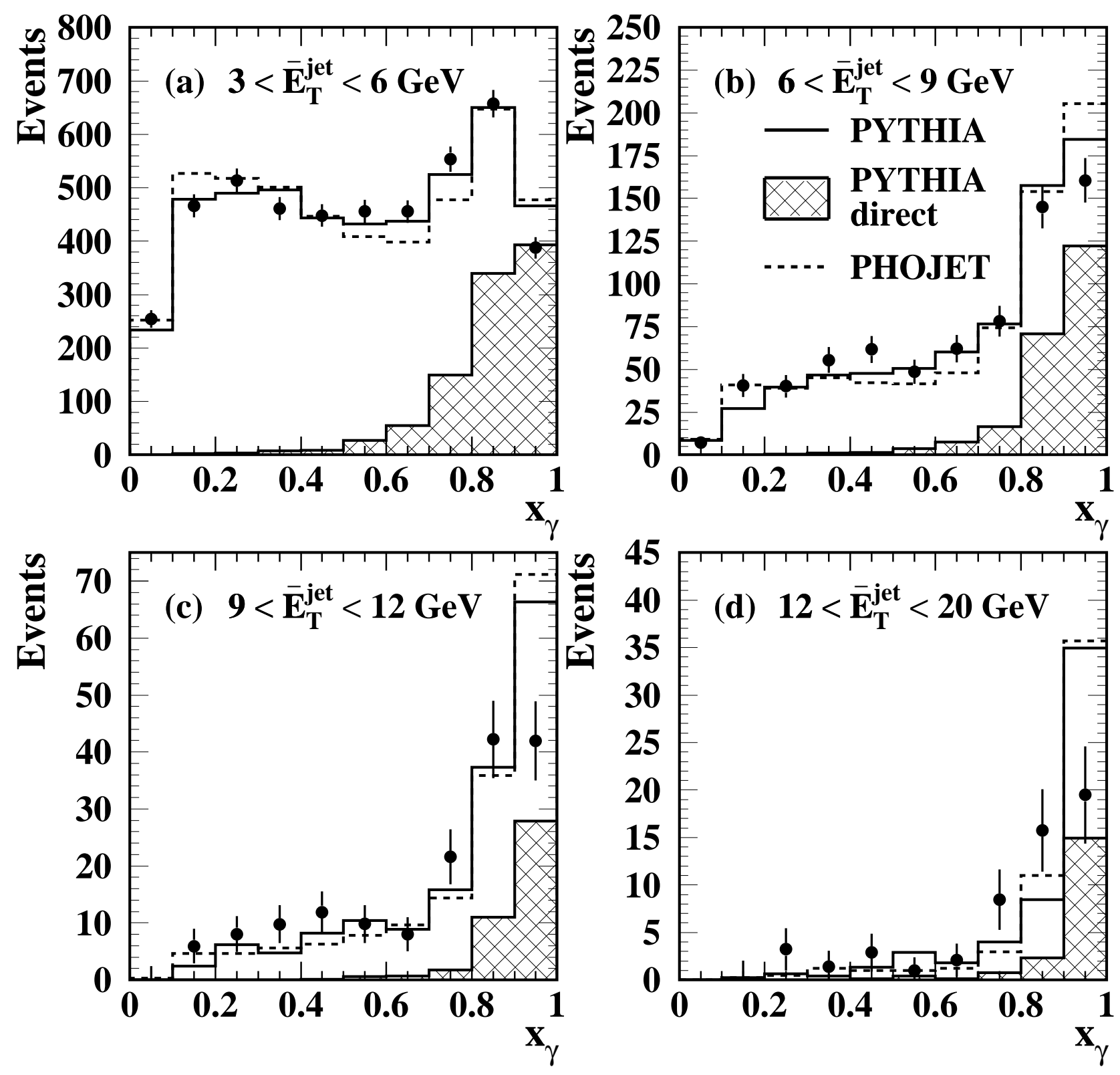

Figure 1: Uncorrected $x_{\gamma}$ distribution in bins of the mean value of $\bar{E}_{\mathrm{T}}^{\text {jet }}$, where $\bar{E}_{\mathrm{T}}^{\text {jet }}$ is calculated as the mean value of $E_{\mathrm{T}}^{\mathrm{jet}}$ of the two jets with the highest $E_{\mathrm{T}}^{\mathrm{jet}}$. The background has been subtracted using the Monte Carlo. The data points are compared to the predictions of PYTHIA (continuous line) and PHOJET (dashed line). The hatched histogram is the direct contribution to the PYTHIA events. The Monte Carlo histograms are normalised to the number of data events. Statistical errors only are shown. 


\section{OPAL}

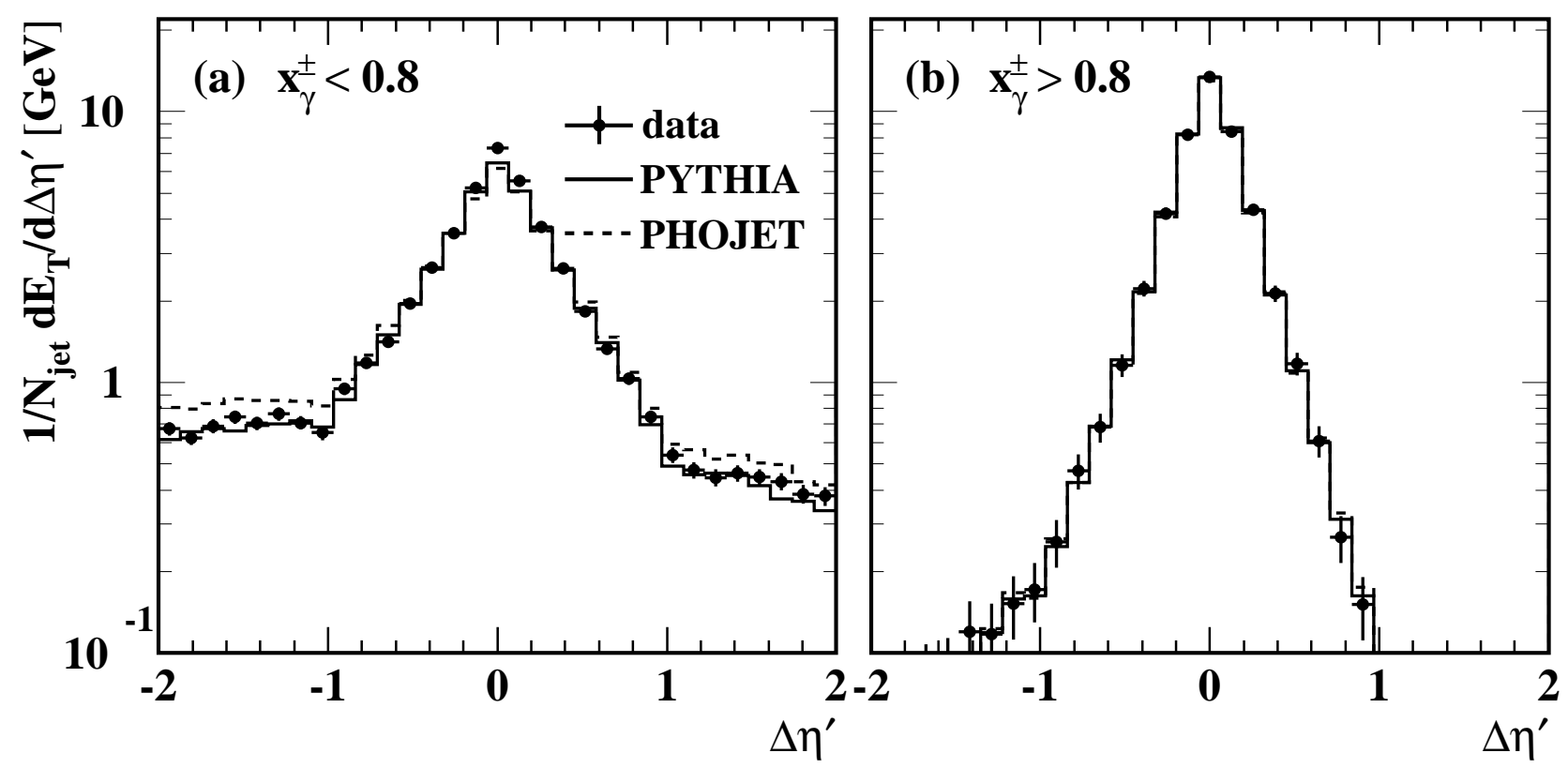

Figure 2: Uncorrected energy flow transverse to the beam direction measured relative to the direction of each jet in two-jet events and normalized to the total number of jets, $N_{\text {jet }}$, in the sample. Jets from data samples with a large contribution of (a) double-resolved and (b) direct events according to their $x_{\gamma}^{+}$and $x_{\gamma}^{-}$values are shown. The energy flow is integrated over $|\Delta \phi|<\pi / 2$. Statistical errors only are shown. The data points are compared to the PHOJET (continuous line) and PYTHIA (dashed line) simulations. 

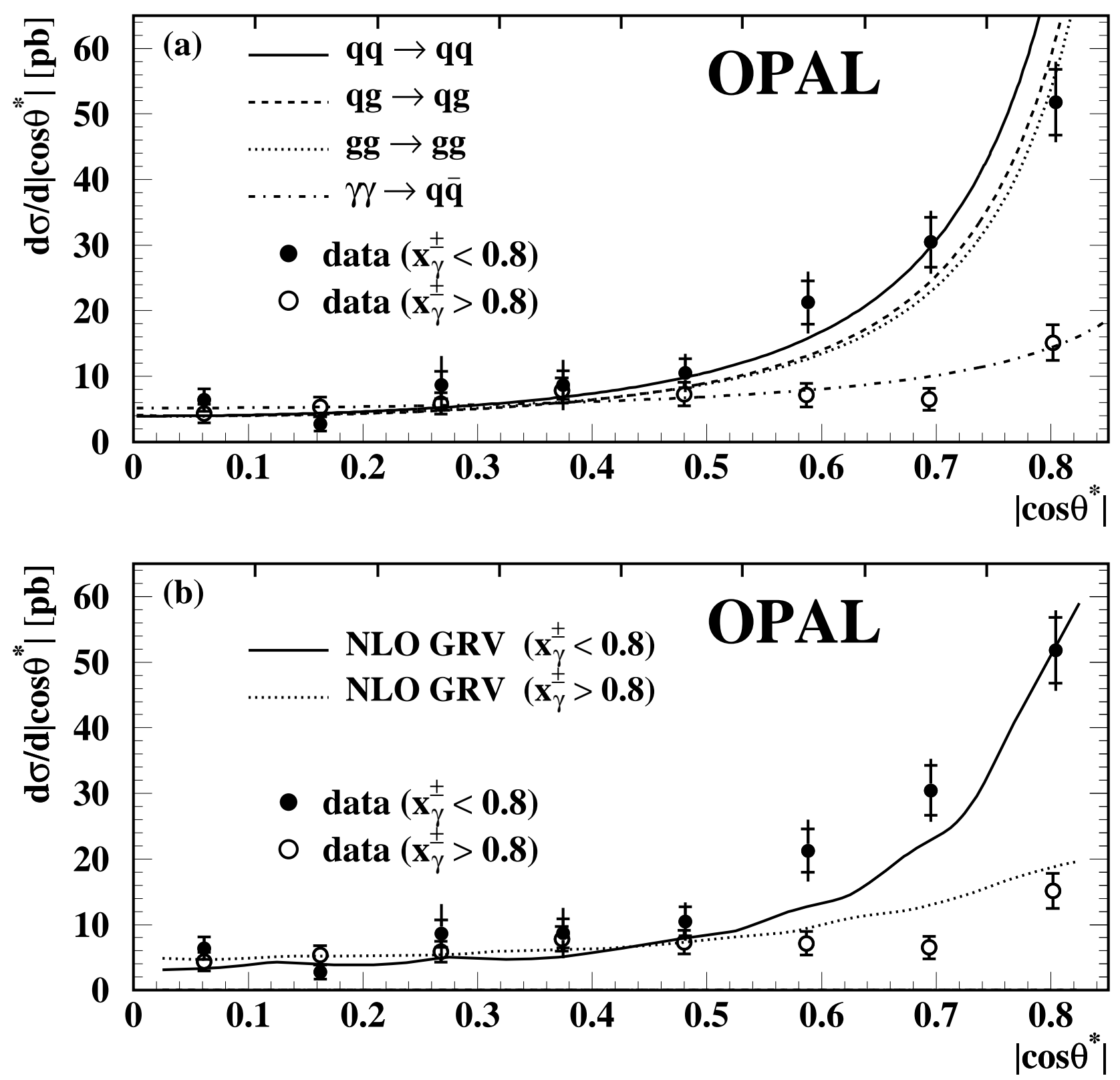

Figure 3: Angular distribution of events with large direct and large double-resolved contributions according to the separation with $x_{\gamma}^{+}$and $x_{\gamma}^{-}$. The data are compared (a) to LO QCD matrix element calculations [6] and (b) to NLO QCD calculations using the GRV parametrisation. The curves are normalised to the data in the first three bins. The open circles show the distribution of events with $x_{\gamma}^{ \pm}>0.8$ and the full circles show the distribution of events with $x_{\gamma}^{ \pm}<0.8$. The inner error bar shows the statistical error and the outer error bar the statistical and systematic errors added in quadrature. 


\section{OPAL}
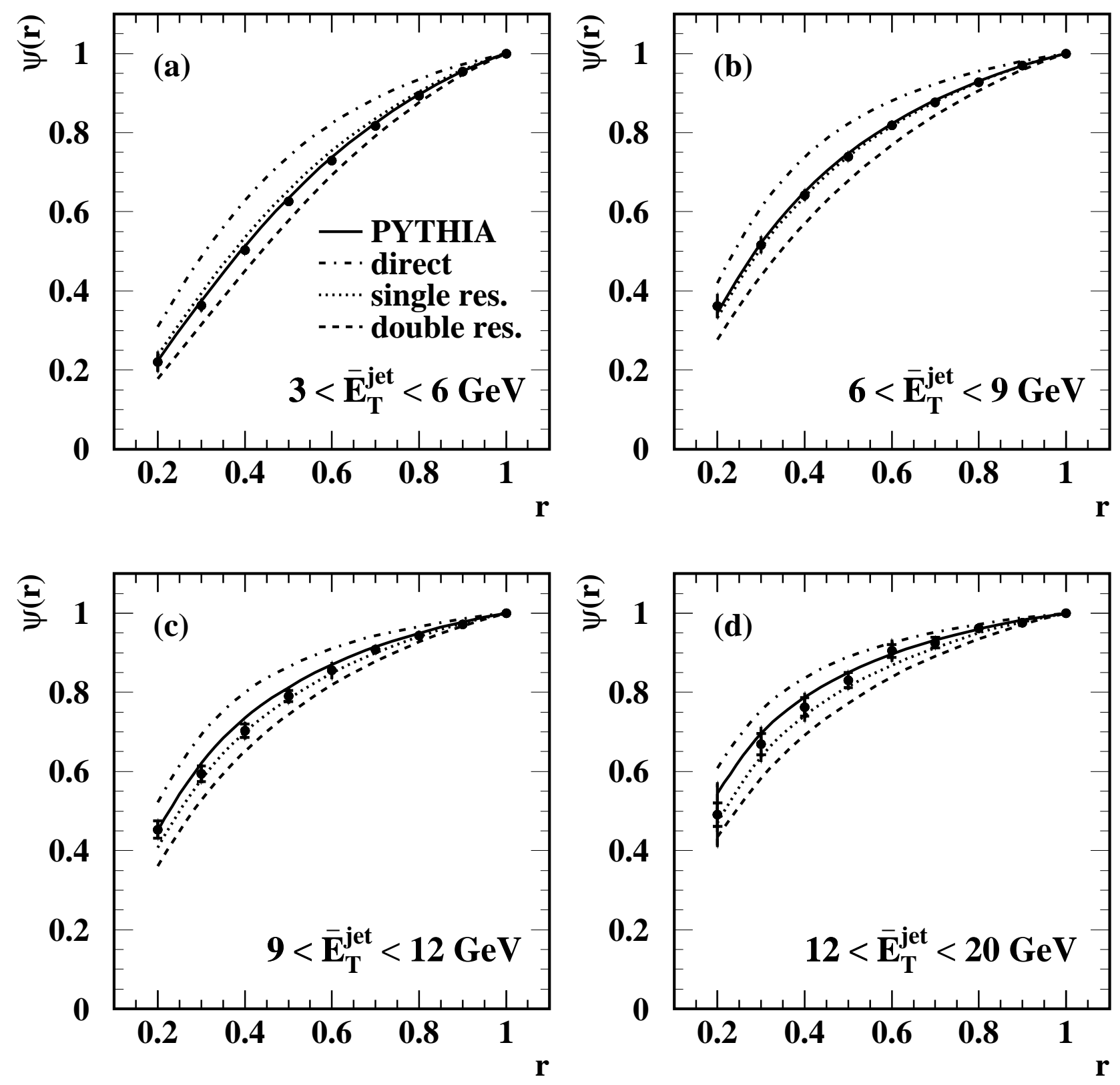

Figure 4: The measured jet shapes, $\psi(r)$, corrected to the hadron level for each of the two highest $E_{\mathrm{T}}^{\mathrm{jet}}$ jets. The jet shapes are shown in bins of $\bar{E}_{\mathrm{T}}^{\mathrm{jet}} ;$ (a) $3<\bar{E}_{\mathrm{T}}^{\mathrm{jet}}<6 \mathrm{GeV}$, (b) $6<\bar{E}_{\mathrm{T}}^{\mathrm{jet}}<9$ GeV, (c) $9<\bar{E}_{\mathrm{T}}^{\text {jet }}<12$ GeV and (d) $12<\bar{E}_{\mathrm{T}}^{\text {jet }}<20$ GeV.The predictions of the direct, singleresolved and double-resolved processes and their sum as predicted by PYTHIA are shown. The inner error bar shows the statistical error and the outer error bar the statistical and systematic errors added in quadrature. 


\section{OPAL}
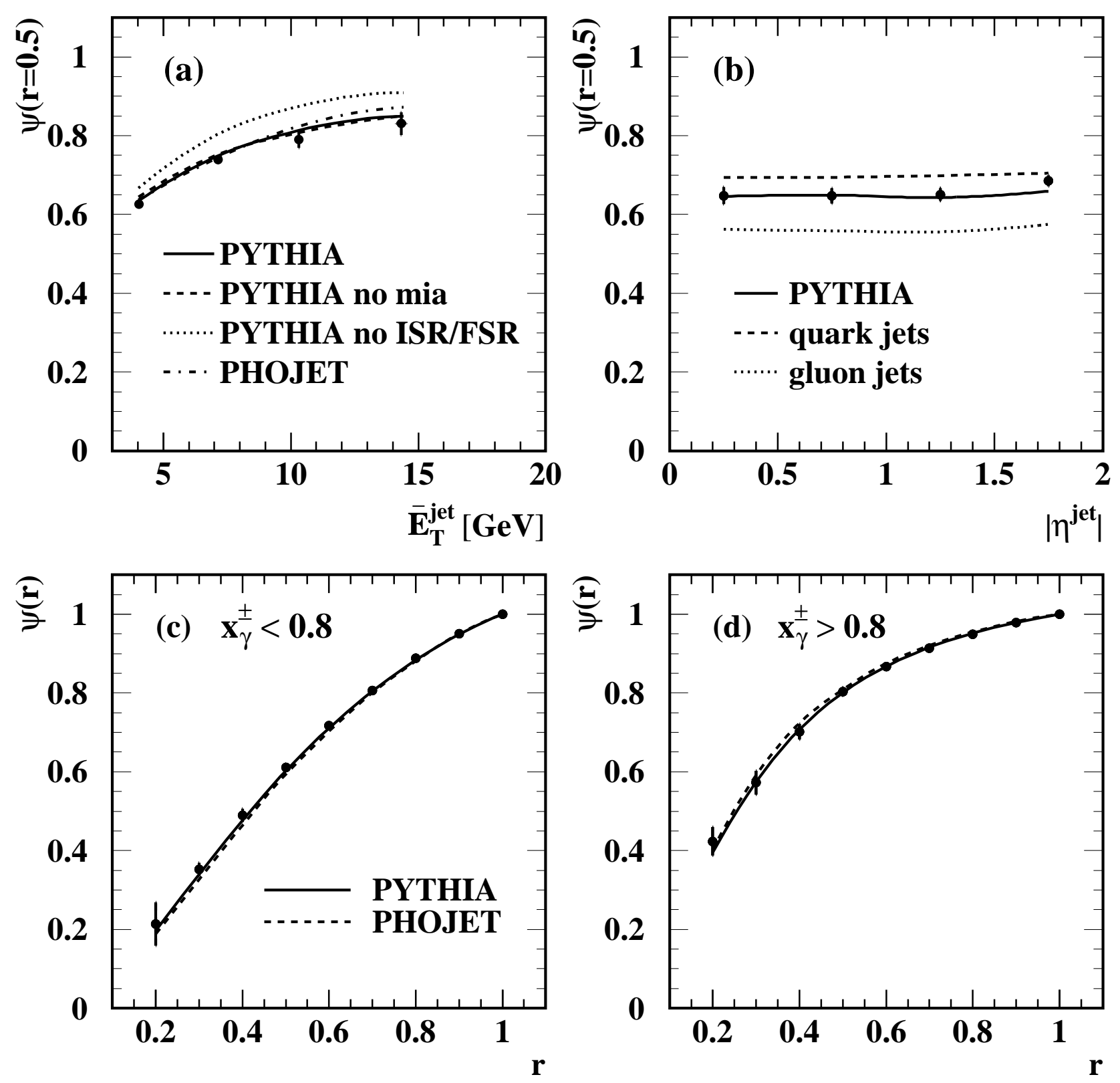

Figure 5: The fraction of the transverse energy of the jets inside a cone of radius $r=0.5$ around the jet axis is shown (a) as a function of $\bar{E}_{\mathrm{T}}^{\mathrm{jet}}$ and (b) as a function of $\eta^{\mathrm{jet}}$. The measured jet shapes corrected to the hadron level, $\psi(r)$, are shown in (c) for $x_{\gamma}^{ \pm}<0.8$ and in (d) for $x_{\gamma}^{ \pm}>0.8$. The statistical error is smaller than the symbol size. The error bars show the statistical and systematic errors added in quadrature. 


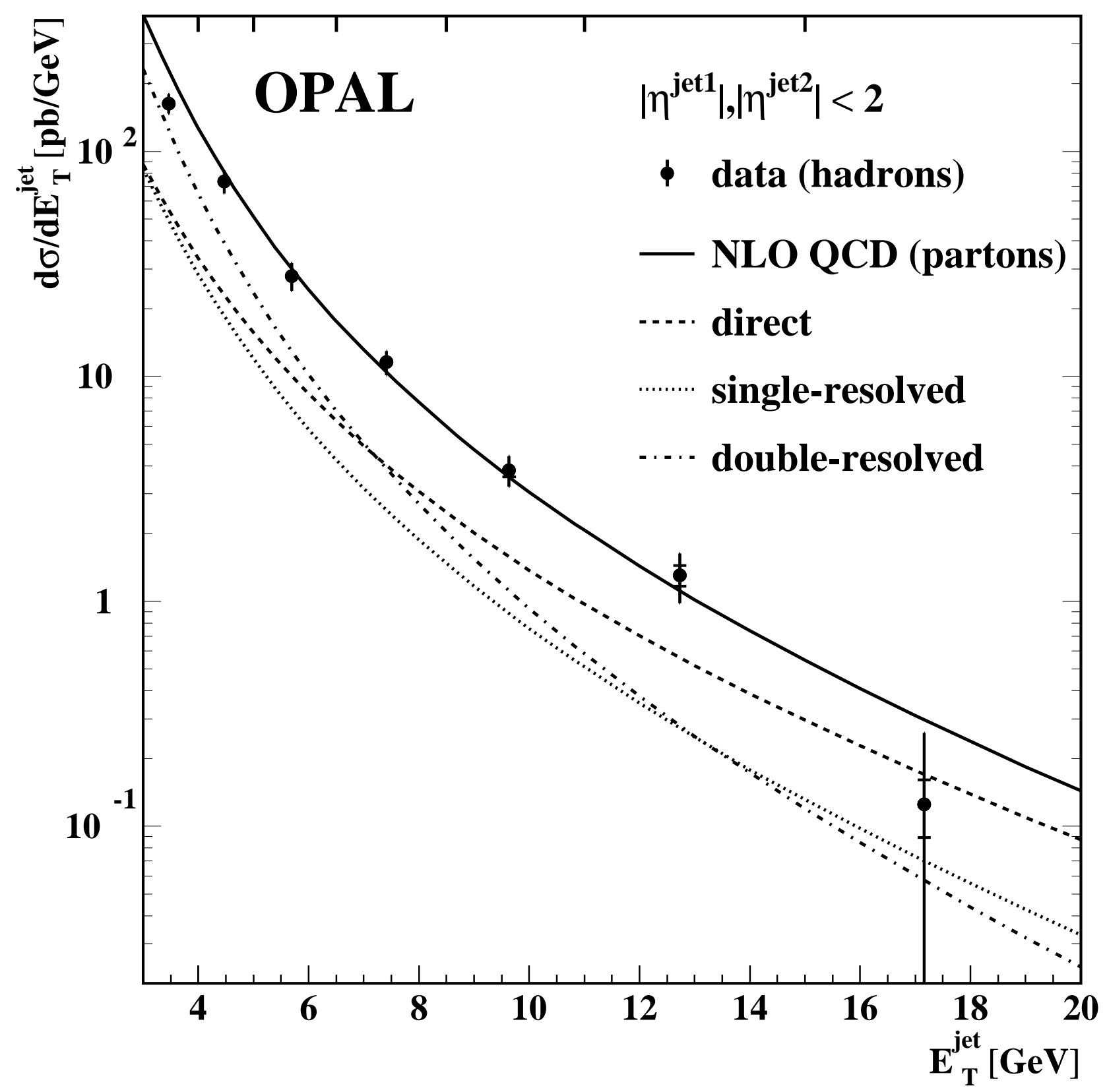

Figure 6: The inclusive two-jet cross-section as a function of $E_{\mathrm{T}}^{\mathrm{jet}}$ for events with $\left|\eta^{\mathrm{jet}}\right|<2$ compared to the NLO calculation by Kleinwort and Kramer [10]. The direct, single-resolved and double-resolved cross-sections and the sum (continuous line) are shown separately. The inner error bar shows the statistical error and the outer error bar the statistical and systematic errors added in quadrature. 


\section{OPAL}

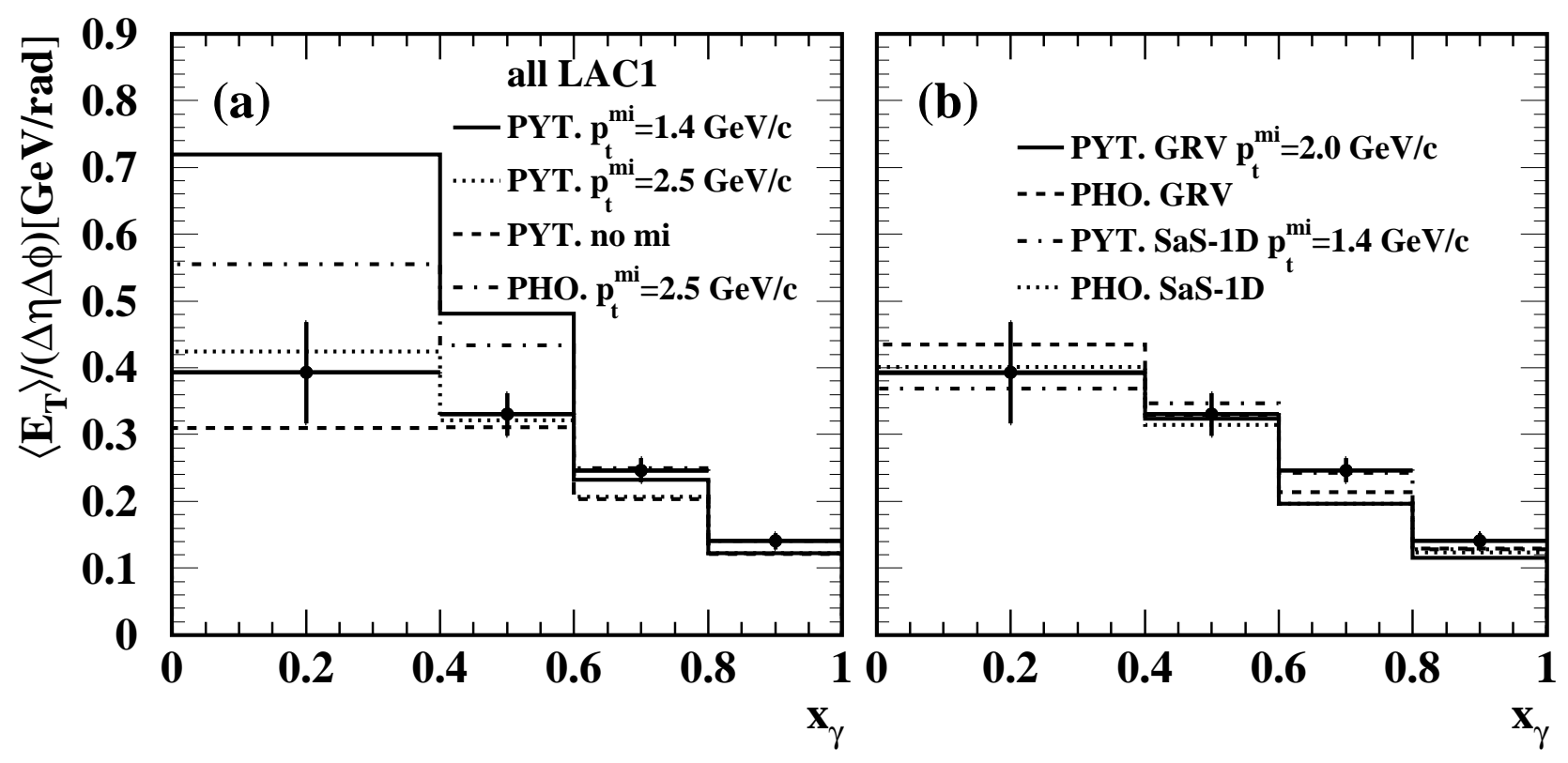

Figure 7: (a) Transverse energy flow outside the jets in the central rapidity region $\left|\eta^{*}\right|<1$ as a function of $x_{\gamma}$. The statistical error is smaller than the symbol size. The error bars show the statistical and systematic errors added in quadrature. 


\section{OPAL}

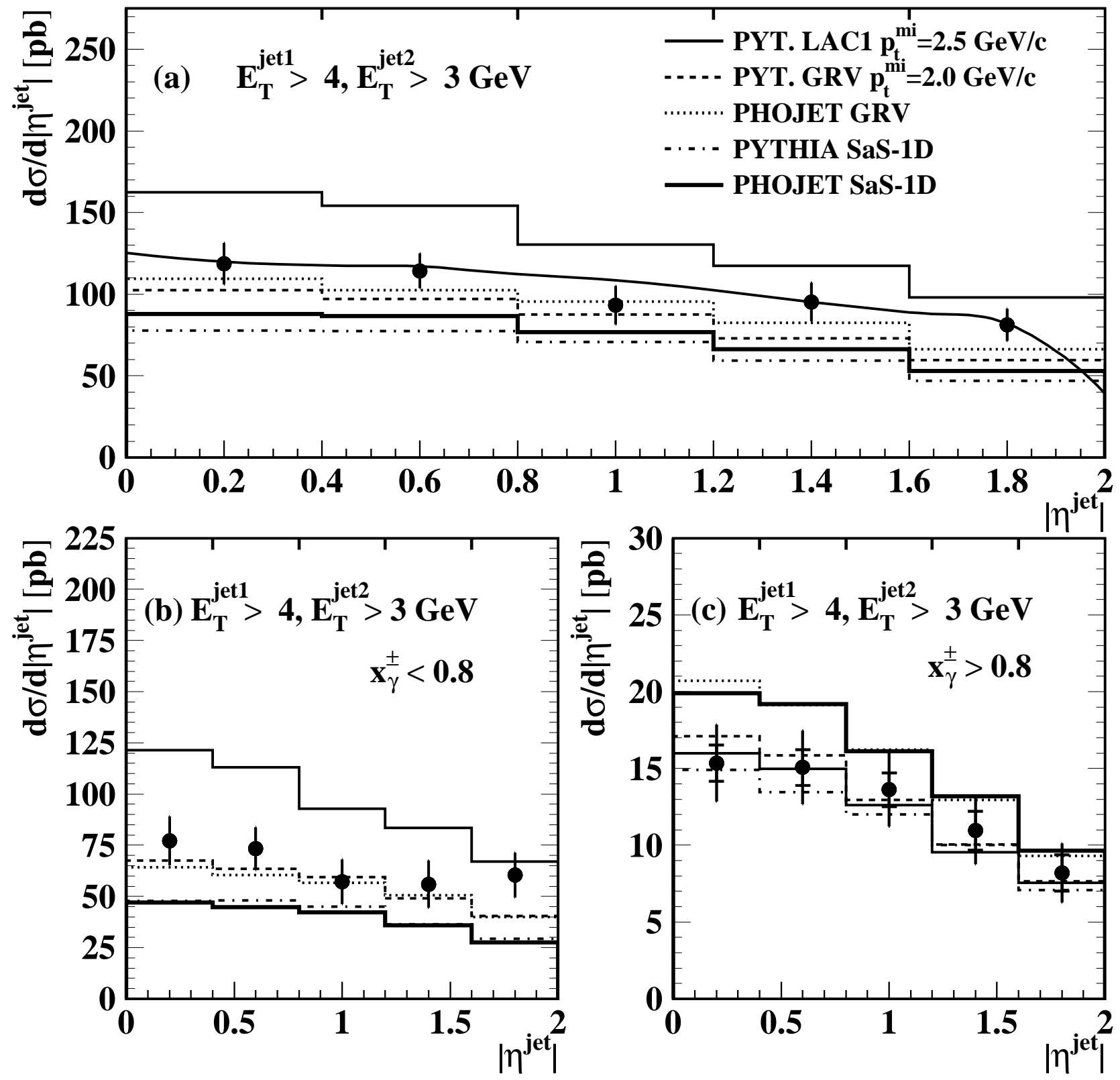

Figure 8: The inclusive two-jet cross-section as a function of $\left|\eta^{\mathrm{jet}}\right|$ for events with $E_{\mathrm{T}}^{\mathrm{jet} 1}>4 \mathrm{GeV}$ and $E_{\mathrm{T}}^{\mathrm{jet} 2}>3 \mathrm{GeV}$ are shown for (a) all events and (b) for events with a large contribution of double-resolved events by requiring $x_{\gamma}^{ \pm}<0.8$ and (c) for events with a large contribution of direct events by requiring $x_{\gamma}^{ \pm}>0.8$. The curve in (a) shows the prediction of the NLO QCD calculation using the NLO GRV parametrisation. The inner error bar shows the statistical error and the outer error bar the statistical and systematic errors added in quadrature. 


\section{OPAL}

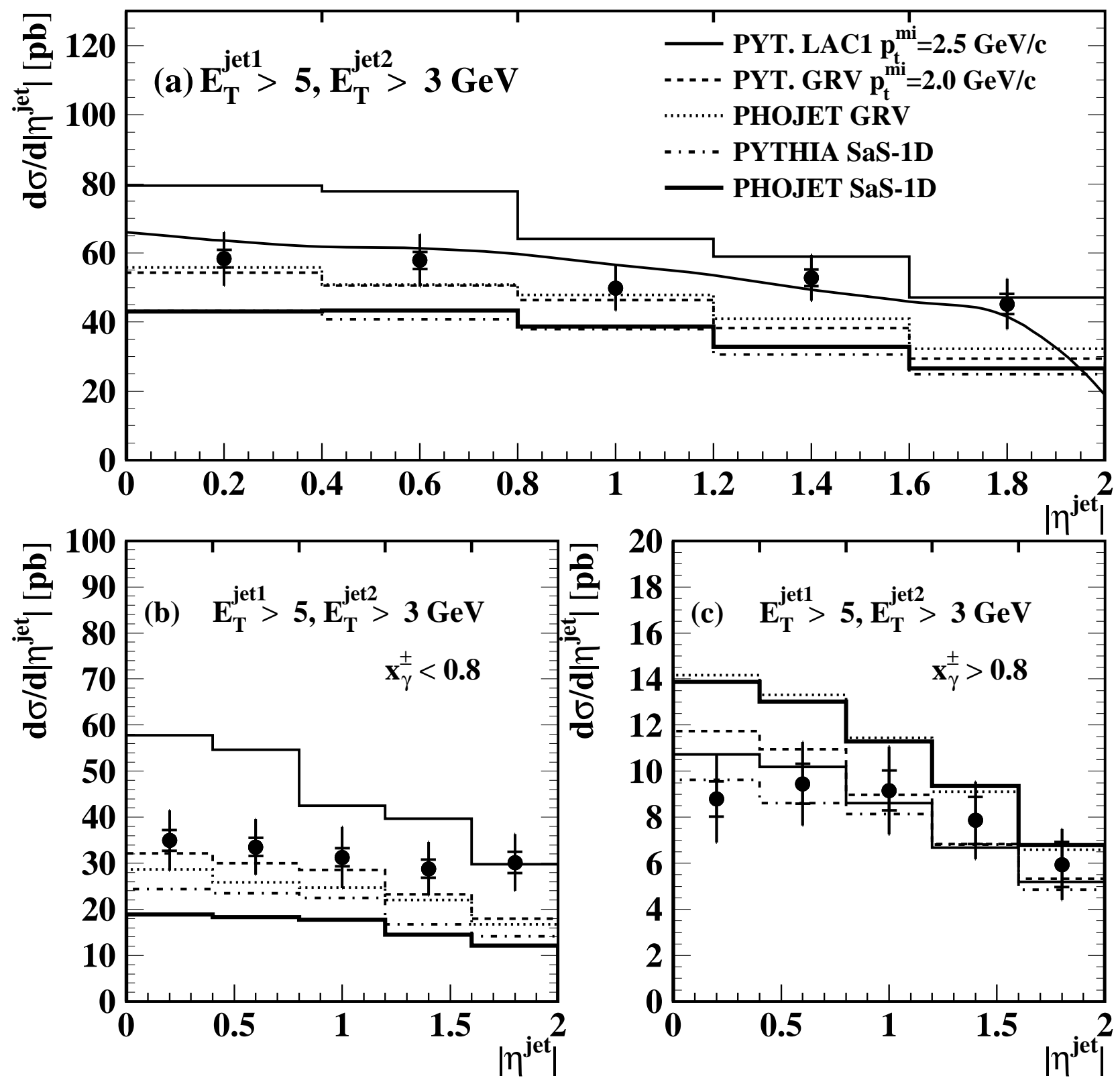

Figure 9: The inclusive two-jet cross-section as a function of $\left|\eta^{\mathrm{jet}}\right|$ for events with $E_{\mathrm{T}}^{\mathrm{jet} 1}>5 G e V$ and $E_{\mathrm{T}}^{\mathrm{jet} 2}>3 \mathrm{GeV}$ are shown (a) for all events and (b) for events with a large contribution of double-resolved events by requiring $x_{\gamma}^{ \pm}<0.8$ and (c) for events with a large contribution of direct events by requiring $x_{\gamma}^{ \pm}>0.8$. The curve in (a) shows the prediction of the NLO QCD calculation using the NLO GRV parametrisation. The inner error bar shows the statistical error and the outer error bar the statistical and systematic errors added in quadrature. 


\section{OPAL}
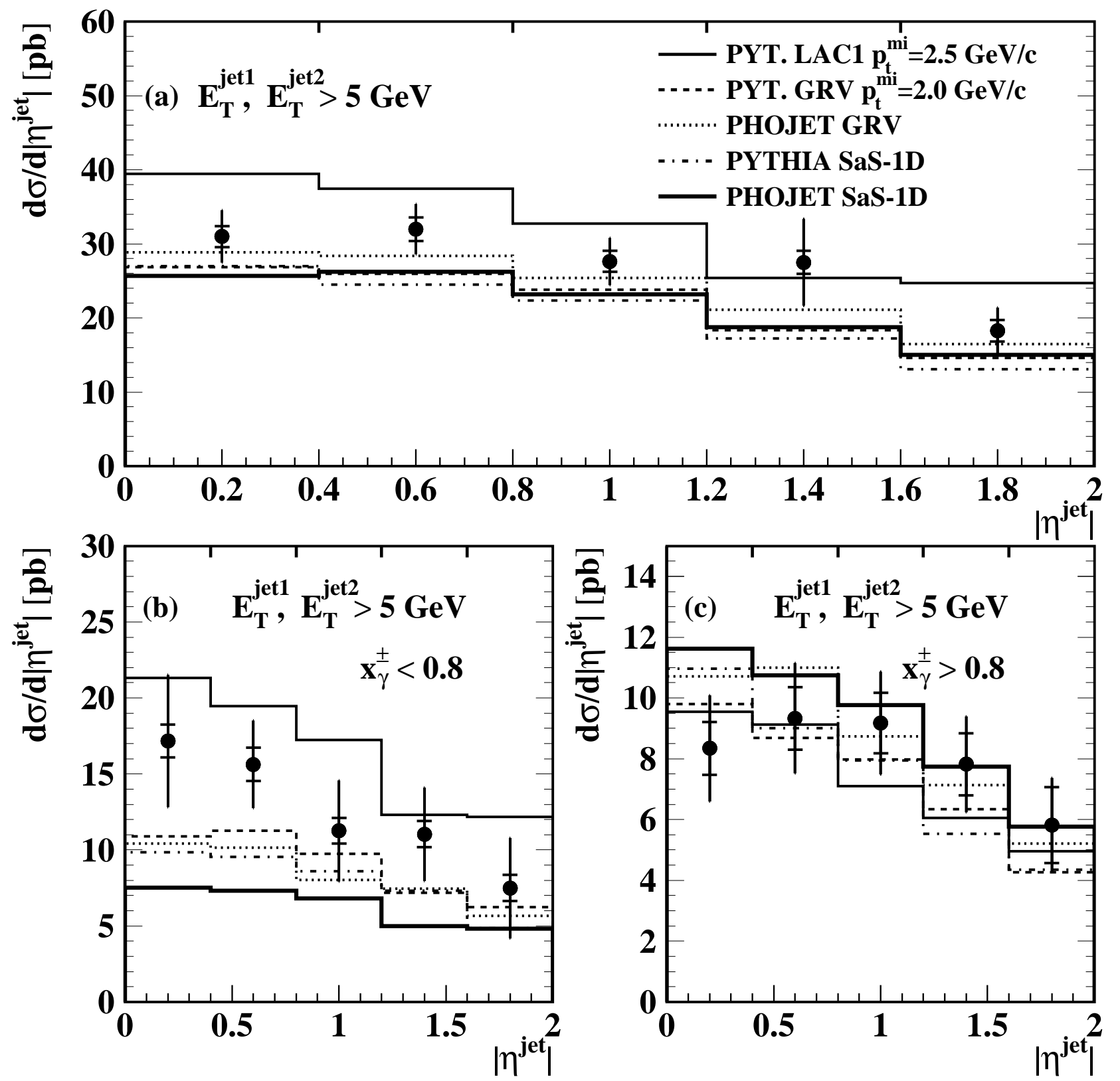

Figure 10: The inclusive two-jet cross-section as a function of $\left|\eta^{\mathrm{jet}}\right|$ for events with $E_{\mathrm{T}}^{\mathrm{jet}}>5 \mathrm{GeV}$ are shown (a) for all events and (b) for events with a large contribution of double-resolved events by requiring $x_{\gamma}^{ \pm}<0.8$ and (c) for events with a large contribution of direct events by requiring $x_{\gamma}^{ \pm}>0.8$. The inner error bar shows the statistical error and the outer error bar the statistical and systematic errors added in quadrature. 

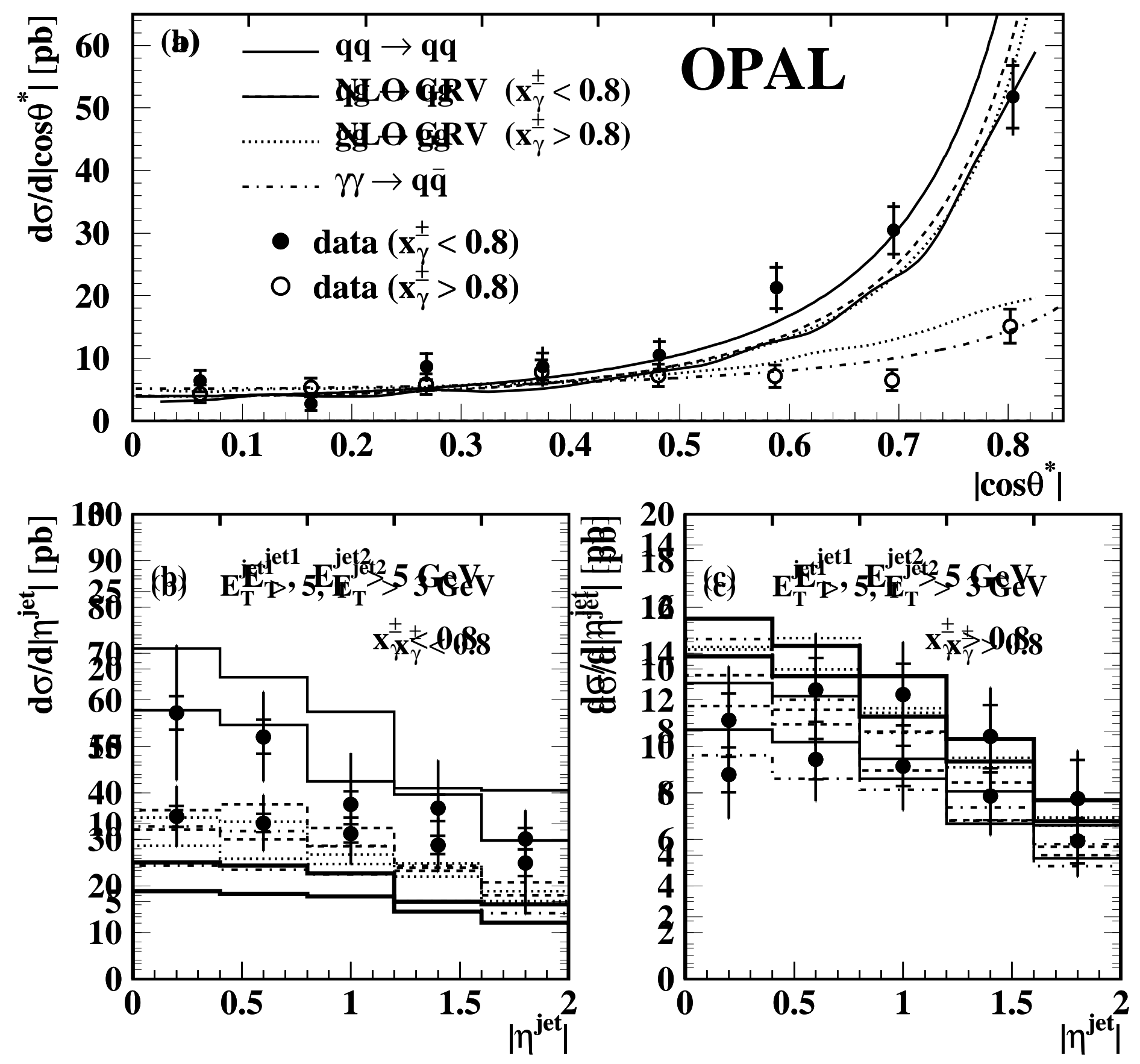